\title{
Bacterial Community in Saline Farmland Soil on the Tibetan Plateau: Responding to Salinization While Resisting Extreme Environments
}

\section{Yiqiang Li}

School of Ocean Sciences, China University of Geosciences (Beijing), Beijing, 100083, China

\section{XUsheng Wang}

School of Ocean Sciences, China University of Geosciences (Beijing), Beijing, 100083, China

\section{Yinghui Chai}

Laboratory division, Eighth Medical Center of Chinese People's Liberation Army General Hospital,

Beijing100000, PR China

\section{Liying Huang}

Institute of Agricultural Quality Standards and Testing, Tibet Academy of Agriculture and Animal Husbandry Sciences,850000, China

\section{Ximing Luo}

Beijing Key Laboratory of Water Resources and Environmental Engineering, China University of Geosciences (Beijing), Beijing, 100083, China

\section{Cheng Qiu}

Institute of Agricultural Quality Standards and Testing, Tibet Academy of Agriculture and Animal Husbandry Sciences, 850000 , China

\section{Qinghai Liu}

Institute of Agricultural Quality Standards and Testing, Tibet Academy of Agriculture and Animal Husbandry Sciences,850000, China

Xiangyu Guan ( $\sim$ guanxy@cugb.edu.cn )

Beijing Key Laboratory of Water Resources and Environmental Engineering, China University of Geosciences (Beijing), Beijing, 100083, China

\section{Research Article}

Keywords: Saline, Tibetan Plateau, Metagenomics, Microbial community, Resistance mechanism

Posted Date: January 20th, 2021

DOI: https://doi.org/10.21203/rs.3.rs-140957/v1 
License: (c) (i) This work is licensed under a Creative Commons Attribution 4.0 International License. Read Full License

Version of Record: A version of this preprint was published at BMC Microbiology on April 20th, 2021. See the published version at https://doi.org/10.1186/s12866-021-02190-6. 
1 Bacterial community in saline farmland soil on the Tibetan Plateau:

2 Responding to salinization while resisting extreme environments

3 YiQiang $\mathrm{Li}^{1}$, YingHui Chai ${ }^{1,2}$, XuSheng Wang ${ }^{1}$, LiYing Huang ${ }^{3}$, XiMing Luo ${ }^{1,4}$,

4 Cheng Qiu ${ }^{3}$, QingHai Liu ${ }^{3}$, XiangYu Guan ${ }^{1,4, *}$

$6 \quad{ }^{1}$ School of Ocean Sciences, China University of Geosciences (Beijing), Beijing,

7 100083, China

$8 \quad{ }^{2}$ Laboratory division, Eighth Medical Center of Chinese People's Liberation Army

9 General Hospital, Beijing100000, PR China

$10{ }^{3}$ Institute of Agricultural Quality Standards and Testing, Tibet Academy of

11 Agriculture and Animal Husbandry Sciences, 850000 , China

$12 \quad{ }^{4}$ Beijing Key Laboratory of Water Resources and Environmental Engineering, China

13 University of Geosciences (Beijing), Beijing, 100083, China

14

$15 *$ Corresponding author: School of Ocean Sciences, China University of

16 Geosciences (Beijing), Beijing, 100083, China. E-mail address: guanxy@cugb.edu.cn 17 (X. Guan). 


\section{Abstract}

21 Background: Soil salinization caused by irrigation will reduce soil health and

22 crop yields. Soil salinization has become one of the world's soil degradation problems.

23 There are few studies on the response of microbial communities to soil salinization in

24 plateau environments. Here, we applied metagenomics technology to make an

25 exploration on the salinized soil microorganisms of the Tibetan Plateau.

26 Results: The metagenomic data results show that the microbial species diversity

27 and genome diversity of saline soil and non-saline soil have changed significantly. we

28 found that the abundances of chemoautotrophic and acidophilic bacteria comprising

29 Rhodanobacter, Acidobacterium, Candidatus Nitrosotalea, and Candidatus

30 Koribacter were significantly higher in saline soil. and the potential degradation of

31 organic carbon in saline soil. The potential degradation of organic carbon in the saline

32 soil, as well as the production of $\mathrm{NO}$ and $\mathrm{N}_{2} \mathrm{O}$ via denitrification, and the production

33 of sulfate by sulfur oxidation were significantly higher compared with the non-saline

34 soil. Both types of soils were rich in genes encoding resistance to environmental

35 stresses (i.e., cold, ultraviolet light, and hypoxia). The resistance of the soil microbial

36 communities to the saline environment on the Tibetan Plateau is based on the

37 absorption of $\mathrm{K}^{+}$as the main mechanism, with cross-protection proteins and

38 absorption buffer molecules as auxiliary mechanisms. Network analysis showed that

39 functional group comprising chemoautotrophic and acidophilic bacteria had

40 significant positive correlations with electrical conductivity and total sulfur, and 
41 significant negative correlations with the total organic carbon, $\mathrm{pH}$, and available

42 nitrogen. The soil moisture, $\mathrm{pH}$, and electrical conductivity are likely to affect the

43 bacterial carbon, nitrogen, and sulfur cycles.

44 Conclusions: These results indicate that the specific environment of the Tibetan

45 Plateau and salinization jointly shape the structure and function of the soil bacterial

46 community, and that the bacterial communities respond to complex and harsh living

47 conditions. In addition, environmental feedback probably exacerbates greenhouse gas

48 emissions and accelerates the reduction in the soil $\mathrm{pH}$. This study will provide insights

49 into the microbial response to soil salinization and the potential ecological risks for

50 the special plateau environment.

51 Keywords: Saline, Tibetan Plateau, Metagenomics, Microbial community, Resistance

52 mechanism

\section{Background}

54 The Tibetan Plateau is located at a high altitude (average $>4,500 \mathrm{~m}$ ), with severe cold

55 and low oxygen levels, and it is strongly affected by ultraviolet radiation $[\mathbf{1}, \mathbf{2}]$. Due

56 to global warming, population increases, and the fragility of the Tibetan Plateau

57 environment, various ecological and environmental problems have occurred, such as

58 vegetation degradation, biodiversity decline, desertification, and salinization [3, 4].

59 Soil is the basis of the function of the global terrestrial ecosystem [5] and soil

60 salinization is considered one of the most pressing environmental challenges for the 
61 world $[6,7]$. The continued salinization of scarce agricultural soil resources will have

62 feedback effects on global climate change, as well as detrimentally affecting the

63 already poor living conditions for people on the Tibetan Plateau. However, the

64 problem of saline soil and its environmental impact in the content of extreme climate

65 change in this complex and fragile environment have received little attention.

66 Soil microorganisms are essential components of the soil ecosystem on the

67 Tibetan Plateau and they play key roles in the health of the ecosystem [8, 9]. Soil

68 microorganisms are involved in the conversion of most nutrients in the soil, and they

69 have critical roles in the decomposition and stabilization of soil organic matter and the

70 nutrient cycle, thereby influencing plant growth and the productivity of aboveground

71 plants $[\mathbf{1 0}, \mathbf{1 1}]$. High salinity has adverse effects on biological activities. The

72 microbial community will adapt to changes in salinity by adjusting its composition

73 and enhancing interactions [12, 13]. Microorganisms adapt to high salinity

74 environment mainly through two mechanisms comprising the synthesis or absorption

75 of organic osmotic agents, and absorbing $\mathrm{K}^{+}$and other inorganic ions to resist osmotic

76 stress $[\mathbf{1 4}, \mathbf{1 5}]$, thereby maintaining the normal life activities of cells under high

77 osmotic pressure conditions. Soil samples from different high salinity regions vary

78 greatly in microbial community structures, and bacteria are more sensitive than fungi

$79[\mathbf{1 6}, \mathbf{1 7 ]}$. In-depth investigations of changes in the structure and function of bacterial

80 communities as sensitive factors will help us to understand the mechanisms

81 responsible for maintaining the function of saline soil ecosystems. 
83 important effects on the microbial community composition and metabolic functions.

84 Salinity leads to significant decreases in the soil microbial diversity and biomass,

85 reductions in the soil enzyme activities $[18, \mathbf{1 9}]$, inhibition of bacterial growth and 86 respiration [20], retardation of the organic matter degradation rate and suppression of 87 nitrification [21]. The mechanisms of bacteria resisting high salinity environments 88 consume large amounts of energy, and the organic matter in the soil will be consumed 89 rapidly [22]. Bacteria with autotrophic capacities have survival advantages in a 90 nutrient-poor environment, thereby leading to changes in the metabolic functional 91 network for the bacterial community. However, no bacteria are specifically adapted to 92 high-salinity soil environments and it is not easy to find bacterial indicator in salinity 93 soil [23]. The soil microbial community on the Tibetan Plateau has responded to 94 extreme environmental pressures via a unique metabolic mechanism [24, 25]. 95 However, the microbial communities in saline soils at high altitude have not been 96 investigated. In the $6^{\text {th }}$ century $\mathrm{BC}$, humans mainly settled in the northeast area of the Tibetan 98 Plateau, and they did not extend their agricultural activities to the land higher than 993600 meters above sea level in the central and southern Tibetan Plateau until 3500 cal 100 yr B.P [26]. The melting of glaciers and repeated freezing-thawing of permanently 101 frozen soils caused by global warming have partially exposed the glacier-covered 102 mineralized rock layers on the surface of the Tibetan Plateau. In addition, the 
103 increased water yield has accelerated the leaching of various minerals in the rock and

104 acid rock drainage into the rivers $[\mathbf{2 7}, \mathbf{2 8}]$. Thus, irrigation using river water has

105 resulted in large amounts of sulfate and metal ions being applied to land, leading to

106 salinization of the soil in the study area. The environmental challenges encountered

107 by soil bacterial communities in farmland in the study area include high soil salinity,

108 temperature differences between the day and night, extremely strong ultraviolet

109 radiation, limited oxygen, and other extreme conditions. Thus, bacterial survival

110 under these conditions evolved specific survival strategies. In this study, we will focus

111 on the: (1) characteristics of bacterial community in saline soil on the Tibetan Plateau,

112 and their biogeochemical cycling processes, (2) the mechanisms associated with the

113 responses to multiple environmental pressures, and (3) the potential impacts of

114 bacterial communities in salinized soil on environmental climate.

\section{Methods}

\section{Soil sampling}

117 The study area is located in Naidong County, Shannan City, Tibet, with an average

118 altitude of $3560 \mathrm{~m}$. Yala Snow Mountain is a natural snow mountain glacier with the

119 highest altitude in the area of $6647 \mathrm{~m}$ and it is the main water source. The study area

120 has a temperate monsoon plateau climate and the air is thin. The average annual

121 temperature in Naidong County is $8.8^{\circ} \mathrm{C}$, the average annual pressure is $660.4 \mathrm{hPa}$, 
122 the average annual solar radiation is $6018.9 \mathrm{MJ}$, and the average annual precipitation

123 is $383.2 \mathrm{~mm}$.

124 In May 2019, saline soil samples (SA) were collected from farmland near the

125 Zhiqu River that had been planted with barley (Fig. S1), and nonsaline soil samples

126 (CK) were collected as a control from farmland near the Yalong River (Xiangqu) that

127 had also been planted with barley. Five subsamples were collected at each sampling

128 site according to the four corners of a square and the center point, where the side

129 length was about $10 \mathrm{~m}$. The surface 5-20 cm soil layer was collected and each sample

130 was packed in a 50-ml sterile centrifuge tube. The sample tubes were refrigerated with

131 ice packs and returned to the laboratory within 24 hours. Each of the subsamples was

132 passed through a 2-mm sieve in the laboratory to remove any stones and plant debris.

133 The five soil subsamples from the same location were mixed to obtain one sample.

134 The mixed saline soil samples were designated as S1, S2, S3, S4, S5, and S6, and the

135 nonsaline soil samples as N1, N2, N3, N4, N5, and N6. Each soil sample was divided

136 into two parts, and one was stored at $4{ }^{\circ} \mathrm{C}$ for subsequent chemical tests and

137 experiments in the laboratory. The other was kept at $-20^{\circ} \mathrm{C}$ for DNA extraction.

\section{Geochemical analysis}

139 The soil samples were dried at $55^{\circ} \mathrm{C}$ and crushed, before passing through a 2-mm

140 sieve. The soil samples were mixed at a soil: water ratio of 1:5 (w/v), shaken well,

141 and allowed to stand for $48 \mathrm{~h}$. The supernatant was passed through a filter membrane 
with a pore size of $0.45 \mu \mathrm{m}$ to prepare the test solution for the experiments. The soil electrical conductivity (EC) value was measured in a suspension with a soil:water ratio of 1:5 (w/v) using a CLEAN Conductivity Tester (CON30; FC Corporation, California, USA). The soil $\mathrm{pH}$ value was measured in a suspension with a soil:water ratio of 1:5 (w/v) using a $\mathrm{pH}$ meter (PB-10; Sartorius, Goettingen, Germany). The pore-water dissolved nitrate $\left(\mathrm{NO}_{3}{ }^{-}\right)$and sulfate $\left(\mathrm{SO}_{4}{ }^{2-}\right)$ contents were analyzed by ion chromatography (DX-120, DIONEX, Bannockburn, IL, USA) [29]. The Total organic carbon (TOC) content (Calculated as carbon dioxide) was confirmed by using a high-frequency infrared Carbon-Sulfur Analyzer (LECO CS744, LECO Corporation, USA). Total nitrogen (TN) was analyzed using a Eurovector elemental analyzer (Isoprime-EuroEA 3000, Milan, Italy). The available nitrogen (AN) contents were determined with the alkaline digestion diffusion method. The total sulfur (TS) contents were measured using the infrared absorption method after high frequency combustion (High-speed Analyzer HWF-900A, Wuxi, China). Other trace metal(loid)s were analyzed by ICP-MS (ThermoFisher X-series, Franklin, MA, USA) and ICP-AES (TJA IRIS-Advantage, Franklin, MA, USA).

\section{DNA extraction, library construction, and metagenomic}

\section{sequencing}

The total DNA was extracted from each soil sample $(0.5 \mathrm{~g})$ using a PowerSoil DNA Extraction Kit (MoBio Laboratories, Carlsbad, CA, USA). The quantity and quality of 
isolated DNA were evaluated using a NanoDrop spectrophotometer (ND-2000,

163 Thermo Fisher Scientific, Waltham, MA, USA) and agarose gel electrophoresis

164 (Bio-Rad, Hercules, CA, USA), respectively. The extracted DNA was stored at $-20^{\circ} \mathrm{C}$

165 until further analysis, or at $-80^{\circ} \mathrm{C}$ for long-term storage.

166 DNA was fragmented to an average size of about 300 bp using Covaris M220

167 (Gene Company Limited, China) for paired-end library construction. Paired-end

168 library was prepared by using TruSeq ${ }^{\mathrm{TM}}$ DNA Sample Prep Kit (Illumina, San Diego,

169 CA, USA). Adapters containing the full complement of sequencing primer

170 hybridization sites were ligated to the Blunt-end fragments. Paired-end sequencing

171 was performed on Illumina HiSeq3000 platform (Illumina Inc., San Diego, CA, USA)

172 at Majorbio Bio-Pharm Technology Co., Ltd. (Shanghai, China) using HiSeq 3000 PE

173 Cluster Kit and HiSeq 3000 SBS Kits according to the manufacturer's instructions

174 (www.illumina.com)

\section{Bioinformatics}

176 The $3^{\prime}$ and 5' ends were stripped (https://github.com/jstjohn/SeqPrep) and low-quality

177 reads were removed (https://github.com/najoshi/sickle). The software SOAPdenovo

178 (http://soap.genomics.org.cn, Version 1.06) was employed to assemble short reads and

179 K-mers were tested for each sample. The software Scaffolds was employed to gene

180 prediction and annotation after with a length over 300 bp were extracted and broken

181 into contigs without gaps. The software CD-HIT 
182 (http://www.bioinformatics.org/cd-hit/) was employed to all sequences sequence

183 identity ( $90 \%$ coverage) from gene sets with $\geq 95 \%$, and were clustered as the

184 non-redundant gene catalog using. After quality control, the software SOAPaligner

185 (http://soap.genomics.org.cn/) was employed to mapped reads to representative genes

186 with $\geq 95 \%$ identity, and the gene abundances were evaluated in each sample. The

187 software BLASTP (Version 2.2.28+, http://blast.ncbi.nlm.nih.gov/Blast.cgi) was

188 employed to taxonomic annotations by aligning non-redundant gene catalogs against

189 NCBI NR database with cutoff: $1 \mathrm{e}^{-5}$ (e-value). The software BLASTP (Version

190 2.2.28+) was employed to annotation the KEGG pathway search against the Kyoto

191 Encyclopedia of Genes and Genomes database (http://www.genome.jp/keeg/) with an

192 cutoff: $1 \mathrm{e}^{-5}$ (e-value).

\section{Statistical analyses}

194 Trimmomatic software was used to excise primers and for quality filtering with the

195 original metagenomic sequences [30]. MetaPhlan2 software was then used to

196 analyze the data and obtain the classifications for the microbial population with a

197 degree of horizontal precision [31]. The species concentration in each sample type

198 was calculated by comparing the mean and median relative abundances in the saline

199 and nonsaline soil samples. R was used to conduct statistical analyses and to plot the

200 taxonomic information at the genus level. The Shannon diversity index was calculated 
201 using the "vegan" package [32]. The Bray-Curtis dissimilarity between different 202 sample types was calculated using the R package "ecodist" [33].

203 KEGG Orthology (KO) functional profiling of the soil microbiota was performed 204 using assemblies derived from whole-genome shotgun sequencing data. Low-quality 205 reads were first trimmed from raw sequencing data using Trimmomatic. High-quality 206 reads were assembled de novo into contigs using metaSPAdes [34] with the default 207 parameters. Next, we performed gene prediction for these scaffolds using PROKKA 208 V.1.11 [35] and the predicted proteins were assigned to the KO using the Kyoto 209 Encyclopedia of Genes and Genomes (KEGG) Automatic Annotation Server. 210 Trimmed high-quality reads located on the given scaffolds were counted to calculate 211 the abundances of Kos in each sample using the Burrows-Wheeler Aligner [36]. The 212 matrix was normalized by dividing the absolute amount of each functional gene by 213 the total number of reads assigned to functional genes in each sample in order to 214 determine the differential expression of microbial functional pathways in the saline 215 and nonsaline soil samples.

216 The bacterial correlations in the saline and nonsaline soil samples were computed 217 based on the relative abundance of each genus using SparCC with 100 bootstraps to 218 estimate the $p$-values for co-occurrence network analysis. The correlation values $p<$ 2190.05 were retained. The co-occurrence network obtained for the microbial 220 communities in the saline and nonsaline soil samples was visualized with Gephi 
221 (version 0.9.1, https://gephi.org/). The closeness values and eigenvectors were

222 calculated for the nodes to measure the node centralities in each network.

223 Community clustering was conducted based on the Bray-Curtis distance for

224 principal coordinate analysis at the genus level, and ADONIS ("vegan") analysis was

225 performed to assess the similarities between groups and the significance of the

226 differences between groups. Genera variation analysis to contrast the two soil sample

227 types was conducted using the Wilcoxon rank sum test with R. Checks and

228 corrections of the false discovery rate (FDR) were performed using the R program

229 fdrtool.

230 Results and Discussion

231 Soil characteristics of Tibetan Plateau

232 In figure2, Soil geochemical analysis showed that the EC values determined for the

233 saline soil were about $9 \mathrm{ds} \cdot \mathrm{m}^{-1}$, and thus the soils were moderately saline [37]. The

234 EC values of the nonsaline soil were less than $4 \mathrm{ds} \cdot \mathrm{m}^{-1}$. The $\mathrm{pH}$ values were $\sim 4.5$ for

235 the saline soil and $\sim 7.2$ for the nonsaline soil. TOC of saline and nonsaline soil were

236 about $1.2 \%$ and about $4.1 \%$ respectively. The soil moisture contents were about $7 \%$

237 higher in nonsaline soil than the saline soil, possibly because salinization destroyed

238 the physical structure of the soil. The nitrate, sulfate, and TS accumulations were

239 significantly higher in the saline soil than the nonsaline soil, whereas TN and AN

240 were significantly lower in the saline soil. Thus, the saline soil was acidic and the 
241 nutrient nitrogen contents were lower. The long-term accumulation of heavy 242 metal(loid)s resulted in an extremely high Mn content. The levels of heavy 243 metal(loid)s such as $\mathrm{Zn}, \mathrm{As}, \mathrm{Cu}$, and $\mathrm{Cr}$, and metal cations such as $\mathrm{K}^{+}, \mathrm{Ga}^{2+}$, and $\mathrm{Mg}^{2+}$

244 were also significantly higher in saline soil than nonsaline soil (Fig. S2). In general, 245 the saline and nonsaline soil differed significantly in terms of most of the geochemical 246 parameters $(\mathrm{FDR}<0.05$, Fig $\mathrm{S} 2)$. The saline soil had a low $\mathrm{pH}$, high salinity, and low 247 nutrient levels, and it was also affected by the extreme unique climate of the Tibetan 248 Plateau, including cold, hypoxia, and strong ultraviolet radiation. Microorganisms 249 may produce a series of community changes and genetic selection under such 250 environmental conditions.

251 Bacterial community in saline and nonsaline soil of Tibetan 252 Plateau

253 After removing low-quality reads of metagenomic sequencing, the quality control 254 results (Table S1) and predicted open reading frames after assembly (Table S2) 255 showed that the average Shannon index was 5.43 for saline soil and that for nonsaline 256 soil was 5.35 (Table S3). In all samples, bacteria accounted for approximately $98.07 \%$ 257 of the total sequences, archaea accounted for approximately $1.32 \%$, and fungi 258 accounted for only $0.01 \%$. The microbial community was dominated by bacteria, and 259 the proportions of archaea and fungi were extremely low. At the microbial phylum 
261 Gemmatimonadetes, Chloroflexi, and other phyla (Fig. 1a).
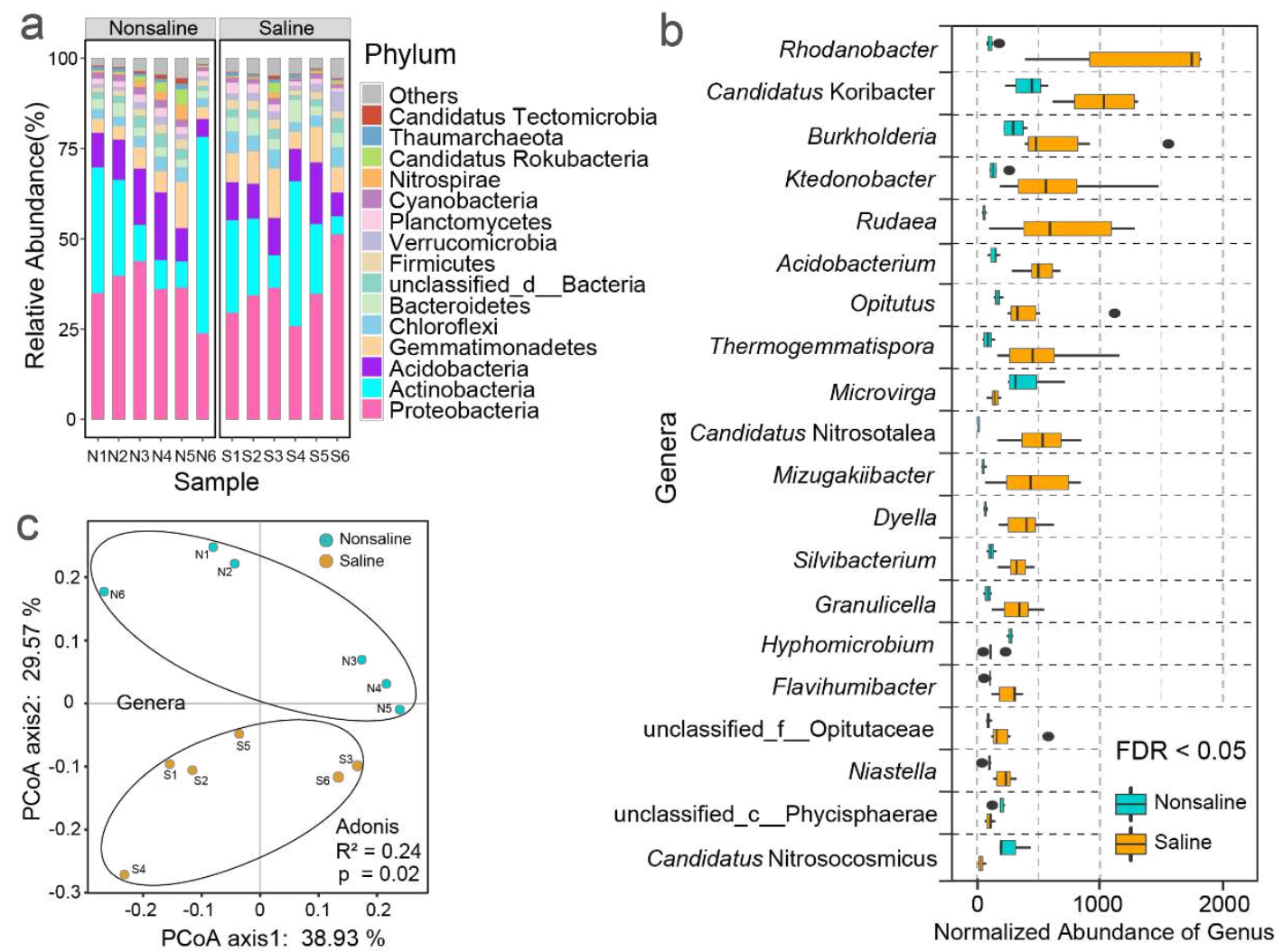

Figure 1. Composition and differences in saline and nonsaline soil on the Tibetan

265 Plateau. a. Bacterial composition at the phylum level in saline and nonsaline soil

266 samples of Tibetan Plateau. b. Top 20 genera with significant differences (FDR < 
274 enriched bacteria in the saline soil had different metabolic strategies. Most were

275 heterotrophic bacteria, but some were chemoautotrophs, such as Rhodanobacter,

276 Granulicella, and Acidobacterium. The dominant significantly enriched bacterial

277 groups associated in the carbon and nitrogen cycles identified in nonsaline soil. For

278 example, Microvirga and Hyphomicrobium have denitrification functions [38, 39],

279 and Candidatus Nitrosocosmicus has ammonia oxidation and carbon fixation

280 capacities [40]. The bacteria in saline soil were found to have specific environmental

281 adaptations (such as chemoautotrophs and obligate acidophilic), whereas the

282 dominant bacteria in nonsaline soil have greater capacity for carbon and nitrogen

283 assimilation. PCoA showed that all of the saline soil samples clustered together and

284 those in nonsaline soil samples formed another cluster, thereby indicating that soil

285 salinization led to significant differences in the bacterial community structure (Fig.

286 1c).

287 The results of the functional abundance based on the KEGG database (Fig. S3a)

288 showed that significant differences in functional genes related to environmental stress

289 resistance (ultraviolet radiation resistance, temperature change response, oxygen

290 limitation response, and salinity adaptation) and important biogeochemical processes

291 (e.g., carbon fixation, nitrogen metabolism, sulfur metabolism, methane metabolism,

292 and heavy metal resistance). PCoA based on the functional composition showed (Fig. 
$293 \mathrm{~S} 3 \mathrm{~b}$ and c) that two separate clusters were formed at both module and KO levels, 294 indicating that salinization also led to significant differences in the soil bacterial 295 functions (ADONIS, $p$-value $<0.05$ ). However, the certain bacterial metabolism 296 related to different element cycles and environmental stress responses should be 297 revealed deeply.

298 Metabolic pathways in saline and nonsaline soil samples of 299 Tibetan Plateau

\section{Carbon cycling}

301 The carbon cycle mainly comprises carbon fixation, carbon degradation, and methane metabolism, which is important for microorganisms in the soil to obtain energy and

303 materials[41]. The carbon fixation pathways (Fig. S4a) in all the samples were mainly

304 composed of the reductive citrate (rTCA) cycle, hydroxypropionate hydroxybutylate 305 (3-HP/4-HB) cycle, crassulacean acid metabolism (CAM) pathway, and Wood306 Ljungdahl (WL) pathway. In Fig. 2, only the abundances of the ackA and folD genes 307 were significantly higher in saline soil than nonsaline soil, and these genes are 308 involved in the 3-HP/4-HB cycle and WL pathway, respectively. Thus, the genes such 309 as $\operatorname{kor} A, s d h A$ and $p p d K$ with significantly higher abundances in saline soil 310 participated in the 3-HP/4-HB cycle and WL pathway, whereas the genes with 311 significantly higher abundances in nonsaline soil mainly participated in the rTCA 312 cycle. The rTCA cycle is prevalent in anaerobic bacteria that are adapted to hypoxic 
313 environments on the Tibetan Plateau and this cycle only requires two ATP equivalents

314 to form pyruvate [42]. The abundance of the WL pathway was probably higher

315 because of its extremely low energy consumption (requirement $<1$ ATP) and

316 requirement for strict anoxic conditions [43]. Thus, low nutritional availability and

317 extreme environments explain why the rTCA cycle and WL pathway predominate in

318 the soil on the Tibetan Plateau, where the 3-HP/4-HB cycle pathway are adaptations

319 to the nutritional deficiencies, respectively.

320

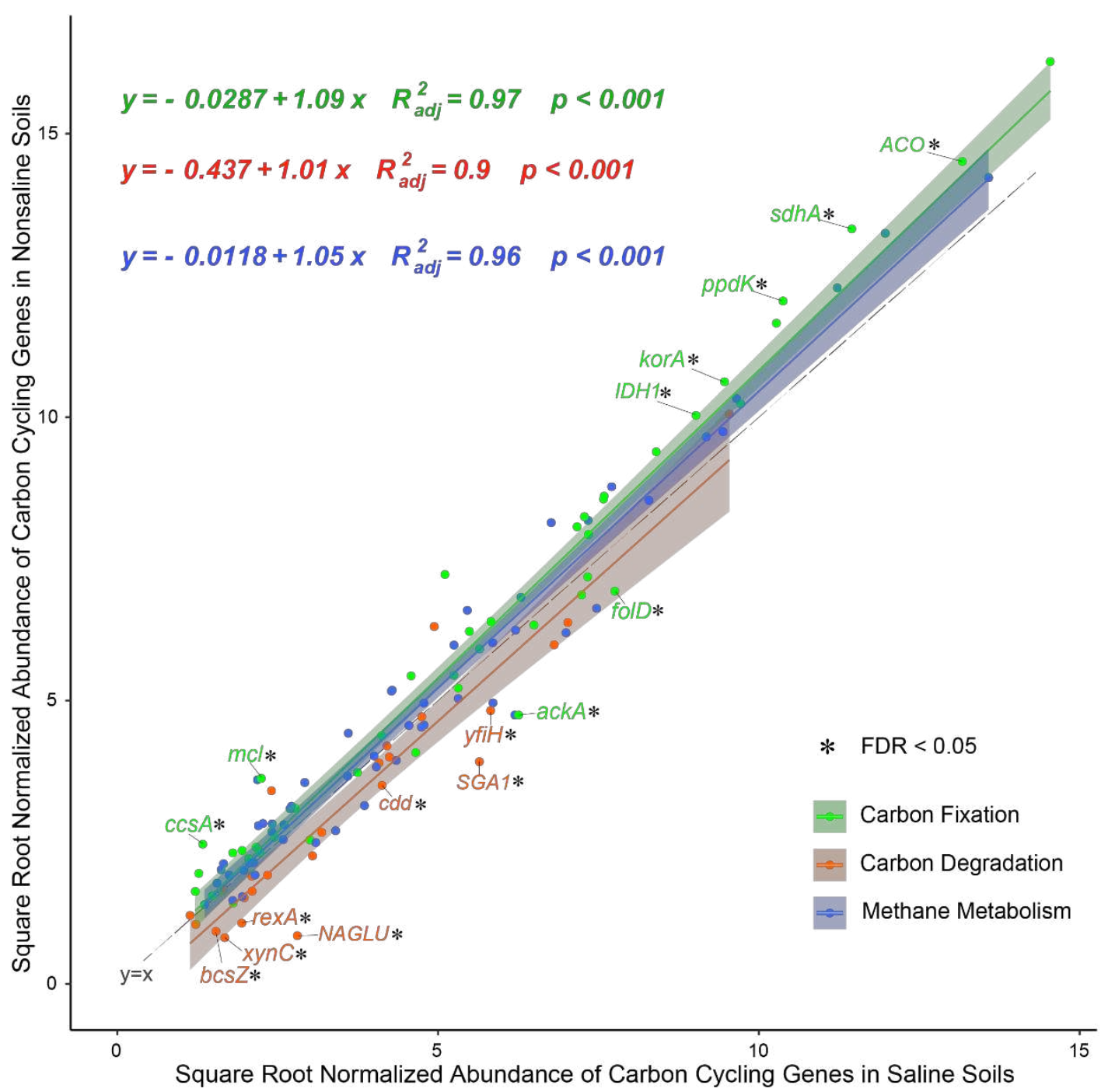



soil on the Tibetan Plateau. The black dotted line equation " $y=x$ " indicates that the horizontal and vertical axes are equal. Green, red, and blue represent genes of carbon fixation, carbon degradation, and methane metabolism pathways, respectively. Genes with significant differences $(F D R<0.05)$ are marked in the corresponding colors and connected with short lines. The total number of reads is normalized to 100000 .

In the carbon degradation pathway (Fig. 2), the genes with significantly higher abundances in saline soil included genes related to starch degradation comprising $c d d$ and $S G A 1$, chitin degradation gene $N A G L U$, lignin degradation gene $y f i H$, cellulose degradation gene $b c s Z$, hemicellulose degradation gene $x y n C$, and $\operatorname{rex} A$, indicating

333 that the potential for carbon degradation was greater in the saline soil. Interestingly, 334 the abundances of genes associated with the degradation of labile carbon (starch, 335 pectin, and hemicellulose) and recalcitrant carbon (cellulose, chitin, and lignin) were 336 higher in the saline soil, possibly because these mechanisms allow microorganisms to 337 maintain their ecosystem functions in the short term in saline soil. In addition, these 338 mechanisms may also explain why the TOC contents were significantly lower in 339 saline soil than nonsaline soil, and the collapse of farmland ecosystems may occur if 340 saline soil remains oligotrophic for a long time $[\mathbf{2 4}, \mathbf{2 5}$. There were no significant 341 differences in the abundances of genes related to methane metabolism in the two soil 342 types (Fig. 2 and Fig. S4b). The lack of significant differences in abundances of genes 
343 related to this process indicates that salinization of the soil had no significant impacts

344 on methane metabolism.

\section{Nitrogen cycling}

346 The nitrogen cycle is one of the crucial soil nutrient cycle processes for the growth of

347 crops and it is driven by soil microorganisms with specific functions [44]. The

348 abundances of genes related to dissimilatory nitrate reduction and denitrification in

349 the soil microbial nitrogen cycle differed significantly in saline and nonsaline soil (Fig.

3503 ), but they did not differ significantly in the nitrate assimilation reduction pathway. 


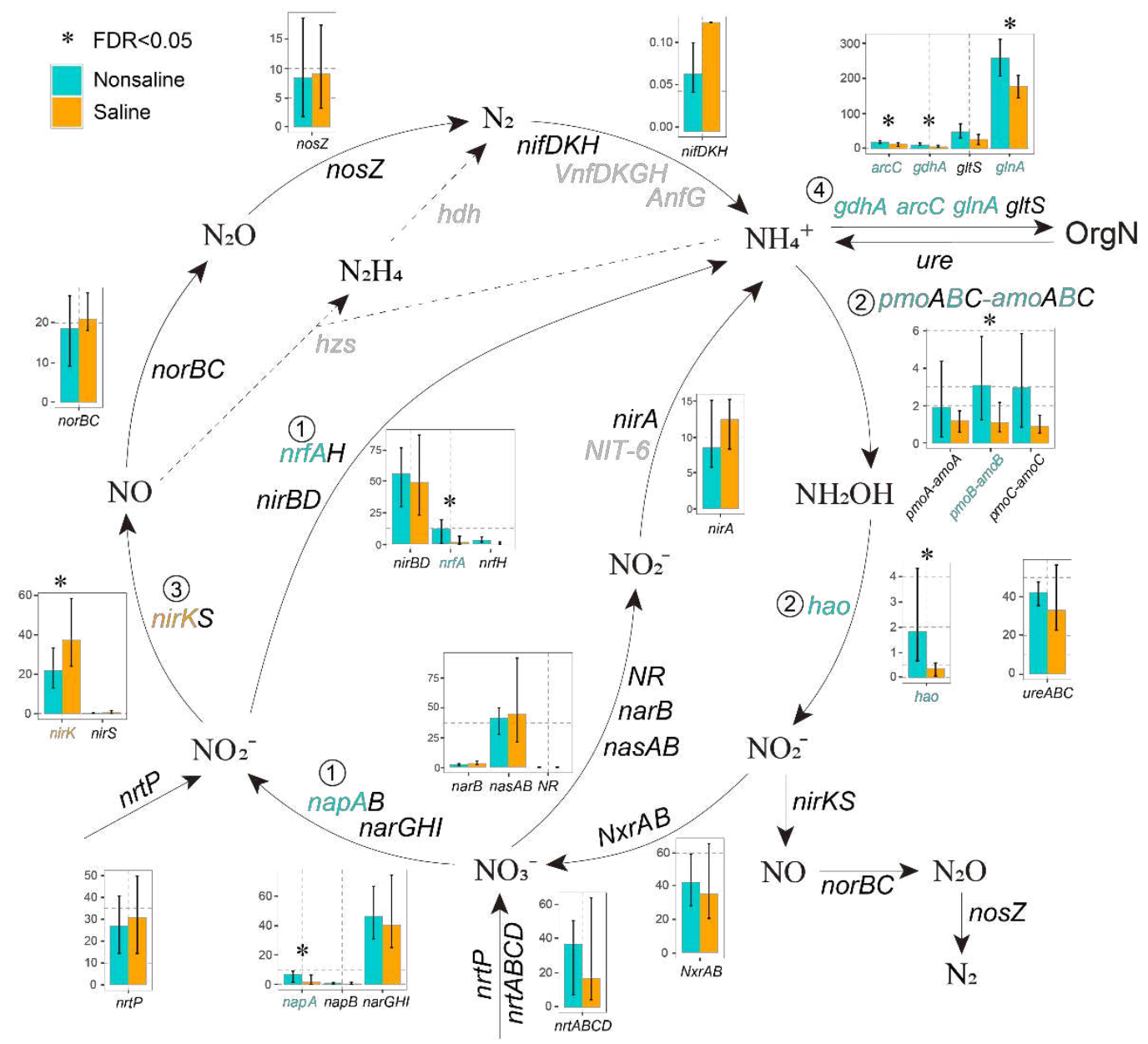

353 Figure 3. Differences in the abundance of genes related to nitrogen cycling in the

354 saline (orange) and nonsaline (cyan) soil on the Tibetan Plateau. Bar plots show

355 the normalized abundances of nitrogen cycling genes. Significantly different (FDR <

356 0.05) genes are marked with “*” and circled numbers. Undetected genes are indicated

357 in gray. Circled numbers identify genes with significant differences: 1, dissimilatory

358 nitrate reduction; 2, nitrification; 3, denitrification; and 4, organic nitrogen conversion.

359 The total number of reads is normalized to 100000 . 
$\mathrm{N}_{2} \mathrm{O}$ produced in saline soil. The abundances of the dissimilatory nitrate reduction genes napA and $n r f A$ were significantly higher $($ FDR $<0.05)$ in saline soil than nonsaline soil (Fig. 3), demonstrating that the potential for ammonia conversion was higher in nonsaline soil. Significantly higher abundances were found in nonsaline soil

371 of the gene $g d h A$ encoding the enzyme that catalyzes the conversion of ammonia to

372 L-glutamate, which are all involved in the conversion of ammonia to glutamic acid 373 (FDR $<0.05)$. It is indicated that more ammonia could be converted into organic 374 nitrogen and this is beneficial for the production of crops. Microorganisms need to 375 synthesize large amounts of amino acids to resist environmental pressure [45]. 376 Therefore, the input of ammonia is critical for the microbial community.

\section{Sulfur metabolism}

378 Due to the high input of sulfate in saline soil, we analyzed the differences in the genes 379 abundance of three pathways related to sulfur metabolism (assimilatory sulfate 380 reduction, dissimilatory sulfate reduction and oxidation, and SOX system) (Fig. 4). 
381 The abundances of genes related to environmental sulfide absorption were 382 significantly lower in saline soil, but the abundances of genes associated with the 383 elimination of toxic intracellular sulfide were significantly higher.

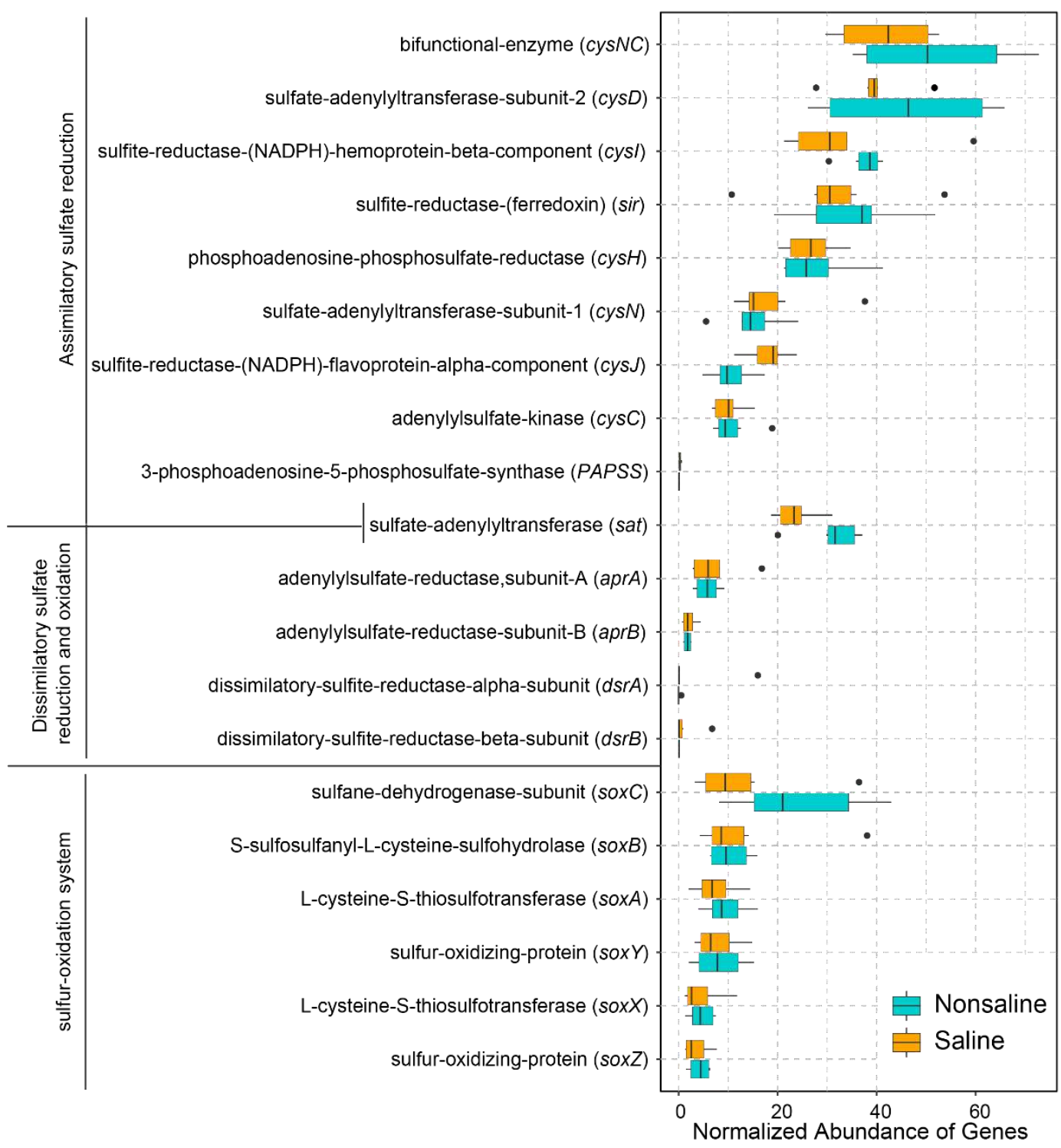

Figure. 4. Difference in abundances of sulfur cycling genes in saline (orange) and normalized to 100000 . 
391 sulfate reduction module that consumes sulfate in the environment and ultimately 392 synthesizes sulfur-containing amino acids. This pathway was probably dominant 393 because of the extremely high sulfate content in saline soil and the large demand for 394 amino acids in the bacterial community. Second, in both soil types, the abundance 395 was low for the dissimilatory sulfate reduction and oxidation module that produces 396 energy and inorganic sulfides, and the abundance of $d \operatorname{sr} A B$ (dissimilatory sulfite 397 reductase) was extremely low, probably because "reverse" sulfite reductase $(d s r)$ is 398 not necessary for the oxidation of sulfide or thiosulfate, and sat (encoding the enzyme 399 that catalyzes the conversion of sulfate to Adenylyl sulfate) and aprAB 400 (adenylylsulfate reductase) participate in the energy production process but they 401 produce cytotoxic sulfites, so their abundances were also low. Finally, the abundance 402 of the $s o e B C$ gene encoding the enzyme in the SOX system that catalyzes the 403 conversion of sulfite into sulfate was significantly higher in saline soil, and this 404 enzyme is important for sulfite oxidation in the cytoplasm (Fig. S5). The oxidation of 405 sulfur can reduce the toxicity of sulfite in cells [46], as well as providing electrons and 406 energy to cells [47].

\section{Metal resistance}

408 In this study, the abundances of heavy metal(loid) resistance genes such as $\operatorname{cop} B, \operatorname{cutC}$, 409 cusRS, and $p c o B$ genes that confer tolerance to copper and the manganese transport 
410 gene $m n t H$ were significantly higher in saline soil (Fig. 5). The differences in the

411 functional genes related to heavy metal absorption showed that the gene ars $B$ related

412 to arsenic absorption had a significantly higher abundance in saline soil (Fig. 5). Both

413 soil types had high abundances of the arsenate reductase gene (arsC), but the

414 abundance of the arsenite oxidase gene (aoxAB) was extremely low because the

415 hypoxic environment promoted the migration of arsenic [48]. The accumulation of

416 arsenite in saline soil on the Tibetan Plateau has toxic effects on microorganisms and

417 crops. However, the abundance of gene arsH (arsenical resistance protein) was

418 extremely low in both soil types (Fig. S6), and thus, the soil bacteria did not have a

419 high capacity to resist the accumulation of arsenic in study area. 


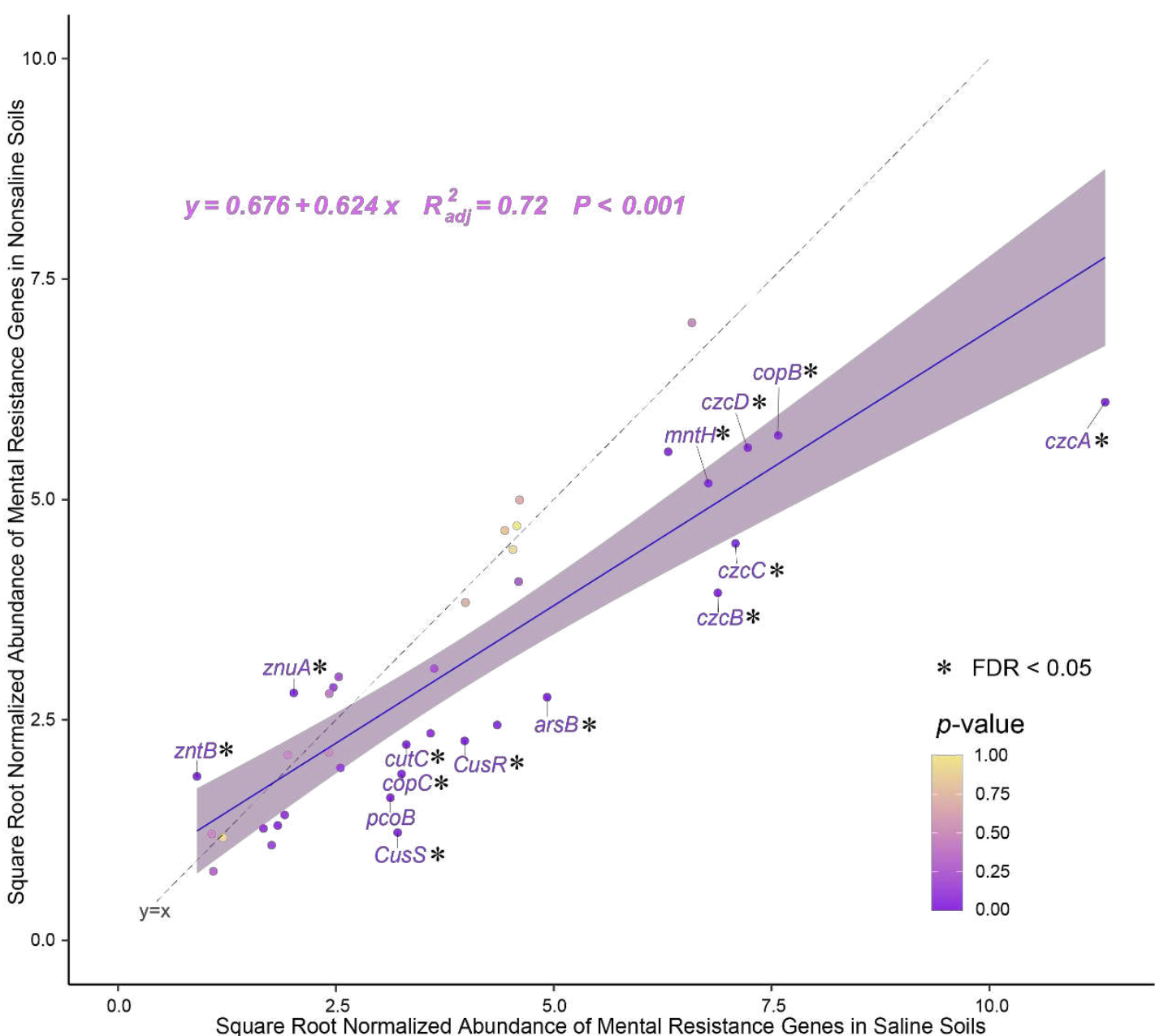

Figure 5. Abundance of heavy metal(loid) resistance genes in saline and lines. The total number of reads is normalized to 100000 .

These results indicate that two main mechanisms mediate microbial resistance to the toxicity of heavy metal(loid)s in saline soils on the Tibetan Plateau, i.e., the efflux of excessive concentrations of heavy metal(loid) ions from cells and expressing 431 proteins that confer tolerance of heavy metal(loid) ions. However, bacteria to resist 
433 increasing concentration of heavy metal(loid) ions in the soil of Tibetan Plateau,

434 which should be studied in future research.

\section{Environmental stress response}

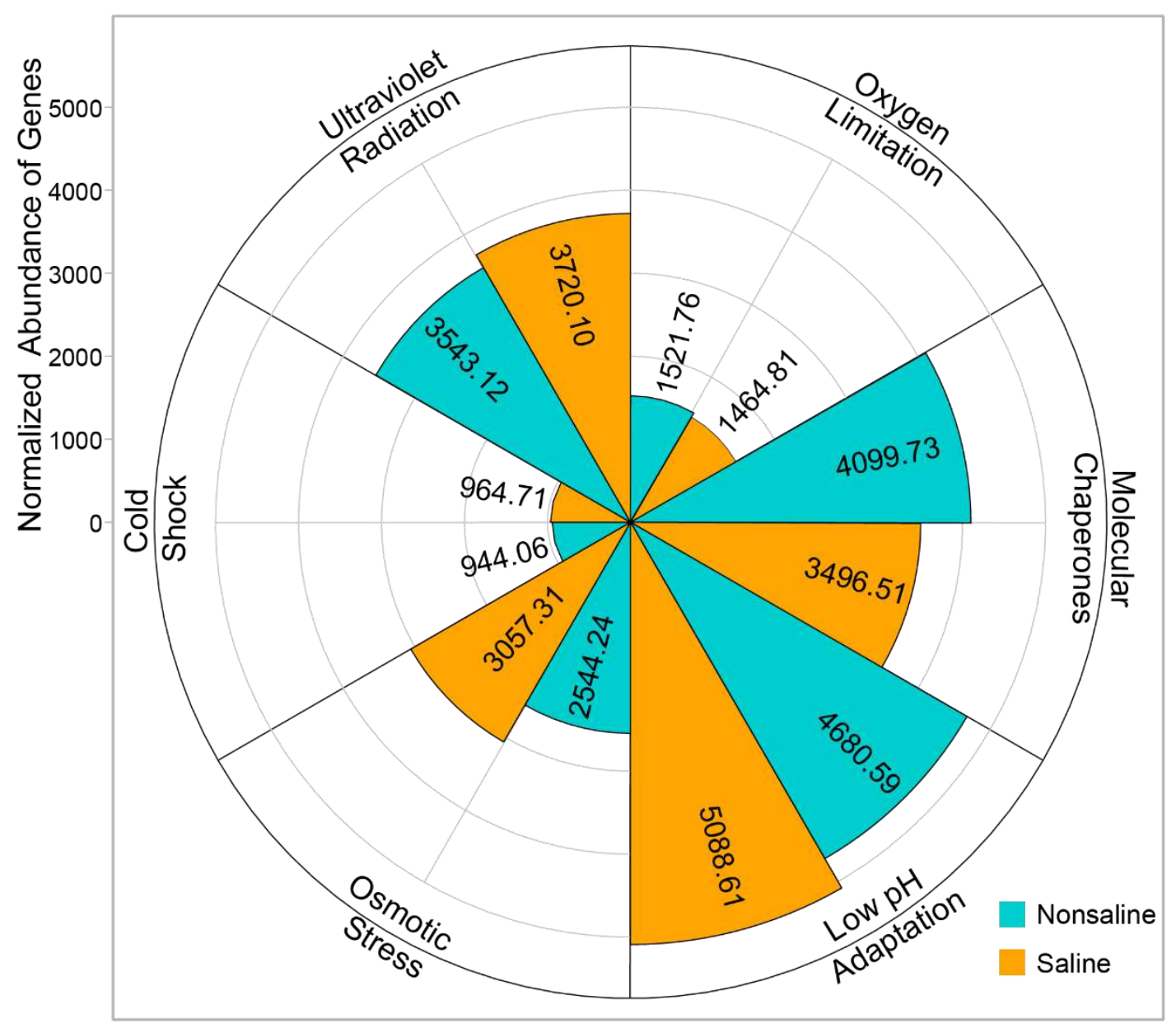

Figure 6. Abundance of the environmental stress resistance genes. The total number of reads is normalized to 100000 .

441 Ultraviolet radiation resistance, the nucleotide excision repair pathway, and 442 photoreactivation (DNA repair via the photolysis enzyme encoded by the $p h r B$ gene) 
444 (Fig. 6). The abundances of the recNO and alkB genes associated with DNA repair 445 were significantly higher in saline soil due to more DNA damage in saline soil (Fig. 446 S8). Bacteria adapt to cold environments mainly through cold shock genes $\operatorname{csp} A$, desK, 447 and $\operatorname{des} R$, which encode enzymes that protect cells from ice crystal damage, and that 448 maintain the transcription and translation processes within cells [50, 51], and via lipid 449 desaturases (desA1, $\operatorname{des} A 2$, and $\operatorname{des} C$; Fig. S7) and the synergy among unsaturated 450 fatty acid synthesis genes $f a b A B$ (anaerobic) and $\operatorname{des} A B$ (aerobic), which are 451 responsible for synthesizing short-chain unsaturated fatty acids embedded in the cell 452 membrane to maintain the cell membrane fluidity and avoid film hardening at low 453 temperatures [52, 53]. These genes were abundant in the two soil types and the 454 abundance of $f a b A B$ was higher than that of $\operatorname{des} A B$ due to low oxygen levels on 455 Tibetan Plateau (Fig. S7). Bateria express large amounts of catalase and peroxidase 456 when responding to oxygen limitation stress [40]. Thus, the abundances of the $c y d B$, $457 f n r$, and $\operatorname{xyy} R$ genes were significantly higher in saline soil (Fig. S7), whereas the katE 458 (catalase) gene was more abundant in nonsaline soil, and the enzyme encoded by katE 459 also acts as a cross-protection protein to help cells cope with environmental pressures.

460 The response mechanisms to oxygen limitation differed between the two soil types.

461 Molecular chaperones help protein folding and refolding to enhance stress resistance 462 [54], and many of these genes had high abundances in the two sample types (Fig. S7). 463 However, the abundances of $d a n K$ and groEL were significantly (FDR $<0.05$ ) higher 
in CK, whereas grpE and $p c c A$ were more abundant in SA, and the abundances of major molecular chaperone genes were higher in nonsaline soil than saline soil (Fig. S7), wich lacked sufficient energy and substrates to synthesize the required molecular 467 chaperones.

In response to high salinity and low $\mathrm{pH}$, the abundance of the $\mathrm{K}^{+}$high-affinity transport system $(k d p A B C)$ was significantly higher in saline soil (Fig. S7). Bacteria generate an inward positive membrane potential through the active inflow of $\mathrm{K}^{+}$to partially deflect the inward flow of protons [55], as well as helping cells to resist the stress due to high osmotic pressure. The metabolism of proton buffer molecules can 473 also maintain the $\mathrm{pH}$ in the cytoplasm, and the abundance of the phosphate uptake 474 gene pstS was significantly higher in saline soil (Fig. S7). Cross-protective genes 475 encoding proteins (osmC, $d p s$, and $k a t E$ ) that maintain the normal life activities of 476 cells under high osmotic pressure were abundant in saline soil, where the abundance 477 of $\operatorname{ssm} C$ was significantly higher. In addition, the microbial self-synthesizing glycine 478 betaine gene $g b s A B$ was more abundant in nonsaline soil, whereas proline and glycine 479 betaine absorption genes (opuABCD, proP, putABP, etc.) were more abundant in 480 saline soil (Fig. S7), probably because the energy consumed by the synthetic permeate 481 was higher than that absorbed from the environment. Therefore, the soil bacteria in 482 saline soil were deficient in substances and energy, so they employed low energy 483 consumption mechanisms to absorb $\mathrm{K}^{+}$, phosphate, and osmotic substances from the 
environment, as well as synthesizing a small amount of protective proteins to resist the low $\mathrm{pH}$ and high osmotic pressure stresses. genes were significantly lower. Genes related to adaptability to the specific climatic conditions on the Tibetan Plateau were abundant and the differences in their

491 abundances in the two soil types were not obvious.

\section{Effects of physicochemical parameters on microbial 493 community and metabolic capacity}

494 Co-occurrence network analysis based on the bacterial genera, functional genes, and 495 environmental factors was constructed in order to understand how environmental 496 factors affect the complex bacterial community structure and functions in saline and 497 nonsaline soils on the Tibetan Plateau (Figs. 7 and S8). The network based on the 498 bacterial community and environmental factors contained three bacterial modules 499 (Table S3). The main negative correlations were found between these three modules, 500 but the bacterial genera within the same module were mainly positively correlated 501 (Fig. 7). 


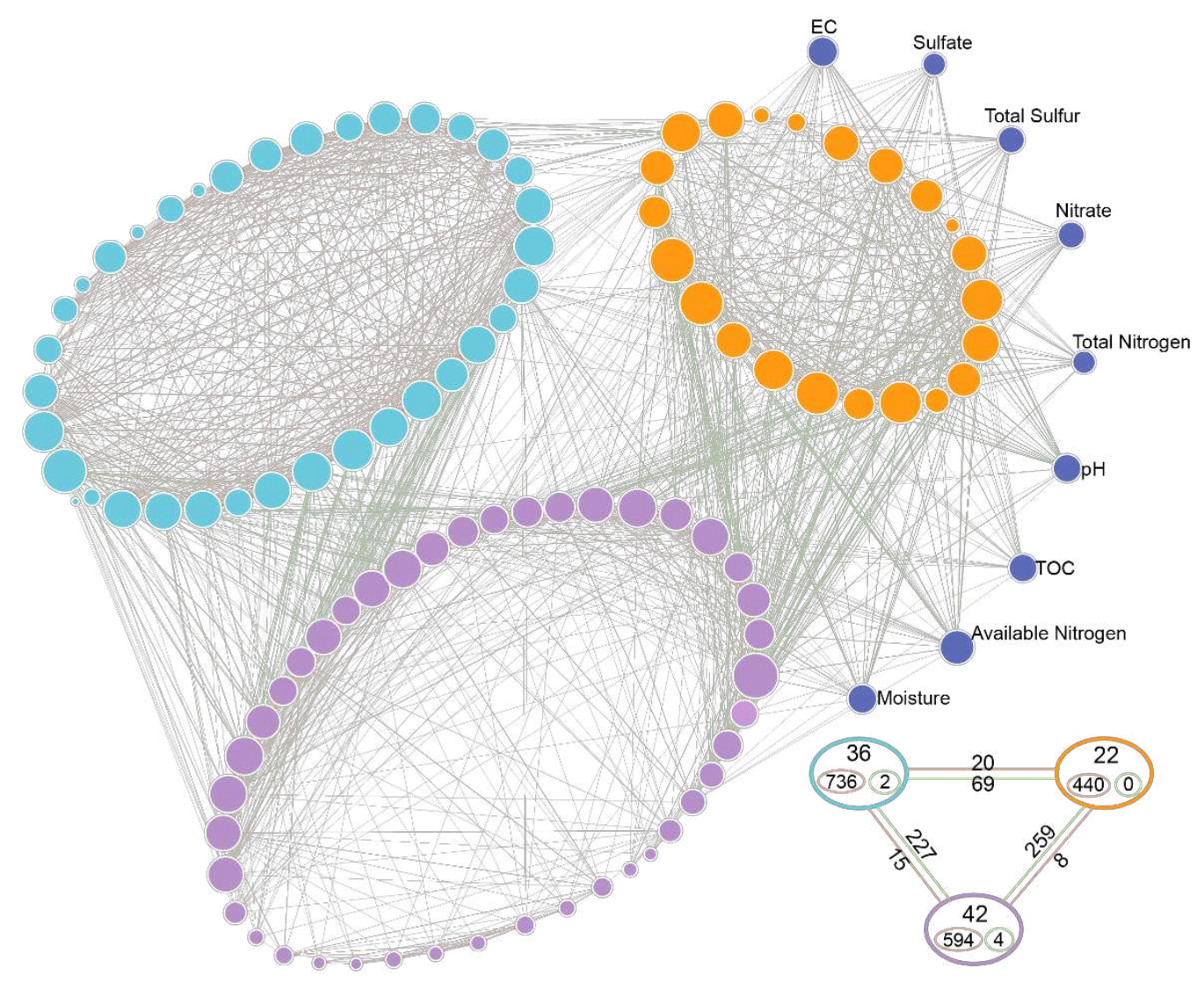

504 Figure 7. Network of the top $\mathbf{1 0 0}$ dominant genera and physicochemical

505 parameters. A connection denotes a Spearman's correlation coefficient with a

506 magnitude greater than 0.6 (positive correlation $=$ red lines) or less than -0.6

507 (negative correlation $=$ green lines) and statistically significant $(p$-value $<0.05)$. The

508 size of each node is proportional to the number of connections (i.e., degree). The

509 thickness of each connection between two nodes (i.e., edge) is proportional to the

510 Spearman's correlation coefficient (i.e., weight), ranging from $|0.6|$ to $|1|$. The network

511 is colored according to the modules, where the nodes clustered in the same module

512 share the same color. The lower right corner is a schematic diagram, and the large

513 circles represent three modules based on network analysis, and the positions are 
514 corresponding. In the large circles, the top number indicates the number of nodes, the

515 red circle and the number indicate positive correlation within the module, and the

516 green indicates negative correlation. Between the large circles, red lines and numbers

517 indicate positive correlations, and green lines and numbers indicate negative

518 correlations.

Module 0 (Orange) contained a total of 22 nodes and most nodes ( $>50 \%)$

521 represented microbial genera with a significant (FDR $<0.05)$ higher abundance in

522 saline soil. Most of the genera are chemoautotrophic and acidophilic bacteria (Table

523 S5 and Fig. 1a), which have strongest correlations with the soil physicochemical

524 parameters, including positive correlations with EC, sulfate, total sulfur, and nitrate,

525 but mostly negative correlations with $\mathrm{pH}, \mathrm{TN}, \mathrm{AN}$ moisture, and TOC (Fig. 7).

526 Module 1 (purple) contained 42 nodes. All of the genera are high abundant in the two

527 soil types. These genera had strong positive correlations with the soil moisture, TOC,

528 and $\mathrm{AN}$, weak positive correlations with $\mathrm{TN}$ and $\mathrm{pH}$, and mostly negative correlations

529 with other environmental factors (Fig. 7). Module 2 (cyan) contained 36 nodes, and

530 these genera were in saline and nonsaline soil. They had very weak negative

531 correlations with $\mathrm{TN}$ and $\mathrm{pH}$, and almost no correlations with other environmental

532 factors. Overall, EC, AN, moisture, TOC, and $\mathrm{pH}$ were the most influential

533 environmental factors. Bacterial community responded to the environmental factors

534 by forming different functional groups (Fig. 7), indicating that the stress due to 
salinity indeed altered the topological roles of microbes and reorganized the keystone populations [56]. The network constructed based on the functional genes and environmental factors

538 in different metabolic pathways (Fig. S8 and Table S4) showed that the main physical 539 and chemical parameters in saline soil (EC, $\mathrm{pH}, \mathrm{TOC}$, moisture, AN, TS, etc.) 540 affected the key biogeochemical cycles for C, N, S, As, and other elements in the soil 541 (Fig. S8). TOC was significantly positively correlated with rTCA cycling which was a 542 high energy efficiency conversion rate pathway in the carbon cycle. However, TOC 543 was significantly negatively correlated with the WL pathway, which may have been 544 related to the extremely low energy consumption and strict anaerobic requirements of 545 this pathway. In addition, TOC, moisture, and $\mathrm{pH}$ were significantly negatively 546 correlated with the main carbon degradation genes (cdd, $S G A 1$, rexA, xynC, bcsZ, and $547 N A G L U$ ), and the high abundance of carbon degradation genes resulted in a 548 significant decrease in the soil TOC concentration (Fig. 3). EC was negatively 549 correlated with carbon fixation genes and positively correlated with carbon 550 degradation genes, indicating that high salinity led to the rapid degradation of organic 551 carbon for the bacterial requirements for substrates and energy. Most of the genes in 552 nitrogen cycle were also positively correlated with $\mathrm{TN}, \mathrm{AN}$ moisture, and $\mathrm{pH}$, but 553 negatively correlated with EC (Fig. S8). In particular, the abundance of gene nirK was 554 significantly negatively correlated with $\mathrm{TN}$, but significantly positively correlated 555 with $\mathrm{pH}$, indicating that the denitrification process in saline soil was affected by 
changes in the soil physicochemical properties, and the long-term accumulation of

557 salinity may have led to the accumulation of nitrate and enhanced denitrification. In

558 addition, sulfate and total sulfur were significantly negatively correlated with the

559 sulfate absorption gene cys $A P U W$, but significantly positively correlated with the

560 sulfur oxidation gene $s o e A B C$, while the key gene $s a t$ in the sulfate reduction pathway

561 was significantly negatively correlated with EC. These results suggest that the

562 accumulation of sulfide in saline have resulted in the bacteria producing more sulfur

563 oxidation genes to synthesize more sulfate. The secondary accumulation of salt would

564 happen in the soil. Moreover, the soil moisture and $\mathrm{pH}$ were negatively correlated

565 with arsenic resistance genes, whereas EC had positive correlations. Overall, high

566 salinity and the accompanying changes in the soil properties had significant impacts

567 on the microbial community and its metabolic network, with significant increases in

568 the chemoautotrophic and acidophilic bacterial modules, as well as effects on other

569 heterotrophic bacteria modules related to the carbon and nitrogen cycles. The bacterial

570 community in the saline soil is likely to consume more soil organic carbon to increase

571 denitrification and intensify the oxidation of sulfur.

\section{Conclusions}

573 Global warming has caused the melting of glaciers and the repeated freezing and

574 thawing of the permanently frozen soil on the Tibetan Plateau, resulting in a sudden

575 increase in the water volume and the exposure of rock formations, where the water 
576 flow has washed over these mineral-rich rock formations into rivers to subsequently

577 increase the soil salinity via irrigation. In this study, we found that the salinization of

578 the soil was accompanied by increased acidity and the accumulation of metal. People

579 who live along the rivers on the Tibetan Plateau are affected by the risk of

580 increasingly saline farming soil. Soil salinization significantly changed the bacterial

581 communities on the Tibetan Plateau, and chemoautotrophic and acidophilic bacteria

582 became dominant. In addition, the key biogeochemical cycle function clusters

583 changed. Carbon degradation, denitrification, and sulfur oxidation gene clusters were

584 highly abundant in saline soils, which were associated with the loss of soil organic

585 matter, increased emissions of $\mathrm{NO}$ and $\mathrm{N}_{2} \mathrm{O}$, and higher sulfate levels in local area.

586 The bacterial community adapting to saline soil did not alleviate the degree of soil

587 salinity, and bacterial community is likely to consume more energy to cope with the

588 extreme climate under saline soil conditions due to the unique features of the Tibetan

589 Plateau. The Yalong River Basin in the study area is a major tributary in the middle

590 reaches of the Yarlung Zangbo River and the most important agricultural area in

591 Shannan City, Tibet. The continuous salinization of agricultural soil along this river

592 will have severe detrimental effects on the local economy and environment. Soil

593 salinization also becomes one of the most direct forms of feedback on the Tibetan

594 Plateau in response to global climate change. 
595 Declarations

596 Ethics approval and consent to participate

597 Not applicable.

598 Consent for publication

599 Not applicable.

600 Availability of data and materials

601 The raw sequence data reported in this paper have been deposited in the Genome

602 Sequence Archive (Genomics, Proteomics \& Bioinformatics 2017) in National

603 Genomics Data Center (Nucleic Acids Res 2021), China National Center for

604 Bioinformation / Beijing Institute of Genomics, Chinese Academy of Sciences, under

605 accession number CRA003771 that are publicly accessible at

606 (http://bigd.big.ac.cn/gsa/s/6AYB1I5n).

607 Competing interests

608 The authors declare that they have no competing interests.

609 Funding

610 This research was supported by the Science and Technology Program of Tibet

611 Autonomous Region (XZ201901NB06 granted to XY Guan), The Fundamental 
612 Research Funds for the Central Universities (2652019077, grant to XY Guan) and

613 National Natural Science Foundation of China (41731282 granted to XY Guan). The

614 authors would like to thank Cidan ZX, Yuxuan X, Minghan W and Yanming Z for

615 their assistance with this study.

\section{Authors' contributions}

617 YL, XG and LH were responsible for collection of samples. XW performed DNA

618 extractions and preparation. $\mathrm{LH}, \mathrm{CQ}$ and $\mathrm{XL}$ measured and analyzed the

619 environmental factors. YL and YC performed sequence assembly, annotation,

620 analysis. QL and YL visualized most of figures. XL and XG developed the project

621 design and provided project oversight. Both YL, XW and XG contributed to the

622 writing of the manuscript. All authors read and approved the final manuscript.

\section{Acknowledgements}

624 The authors thank Xianzhuang Li and Jiaxiang Zhao of Beijing Key Laboratory of

625 Water Resources and Environmental Engineering, China University of Geosciences

626 (Beijing), Beijing, China, for their assistance in soil sample processing and physical

627 and chemical testing.

628

629

630 
632 1. Kang S, Xu Y, You Q, Flügel W-A, Pepin N, Yao T: Review of climate 633 and cryospheric change in the Tibetan Plateau. Environmental $634 \quad$ Research Letters 2010, 5(1).

635 2. Guan X, Wang J, Zhao H, Wang J, Luo X, Liu F, Zhao F: Soil bacterial 636 communities shaped by geochemical factors and land use in a 637 less-explored area, Tibetan Plateau. BMC genomics 2013, 14 (1):820. 638 3. Qiu J: China: the third pole. Nature 2008, 454(7203):393-396.

639 4. Zhang H, Fan J, Wang J, Cao W, Harris W: Spatial and temporal 640 variability of grassland yield and its response to climate change 641

5. Rillig MC, Ryo M, Lehmann A, Aguilar-Trigueros CA, Buchert S, Wulf

6. Cheng G, Wu T: Responses of permafrost to climate change and their

9. Li HY, Webster R, Shi Z: Mapping soil salinity in the Yangtze delta: REML and universal kriging (E-BLUP) revisited. Geoderma 2015, 237-238:71-77.

10. Zovko M, Romić D, Colombo C, Di Iorio E, Romić M, Buttafuoco G, Castrignanò A: A geostatistical Vis-NIR spectroscopy index to assess the incipient soil salinization in the Neretva River valley,

665 11. Peng J, Biswas A, Jiang Q, Zhao R, Hu J, Hu B, Shi Z: Estimating 666 soil salinity from remote sensing and terrain data in southern Xinjiang Province, China. Geoderma 2019, 337:1309-1319. of microbes from saline soils and non-saline soils respond 
similarly to changes in salinity. Soil Biology and Biochemistry 2012, 47:175-178.

13. Zheng W, Xue D, Li X, Deng Y, Rui J, Feng K, Wang Z-1: The responses and adaptations of microbial communities to salinity in farmland soils: a molecular ecological network analysis. Applied Soil Ecology 2017, 120:239-246.

14. Csonka LN: Physiological and genetic responses of bacteria to osmotic stress. Microbiology and Molecular Biology Reviews 1989, $53(1): 121-147$.

15. Zhou M-X, Renard M-E, Quinet M, Lutts S: Effect of NaCl on proline and glycinebetaine metabolism in Kosteletzkya pentacarpos exposed to Cd and Zn toxicities. Plant and Soil 2019, 441(1-2):525-542.

16. Zhang K, Shi Y, Cui X, Yue P, Li K, Liu X, Tripathi BM, Chu H: Salinity is a key determinant for soil microbial communities in a desert ecosystem. MSystems 2019, 4(1).

17. Yu Y, Zhao C, Zheng N, Jia H, Yao H: Interactive effects of soil texture and salinity on nitrous oxide emissions following crop residue amendment. Geoderma 2019, 337:1146-1154.

18. Ma L, Ma F, Li J, Gu Q, Yang S, Wu D, Feng J, Ding J: Characterizing and modeling regional-scale variations in soil salinity in the arid oasis of Tarim Basin, China. Geoderma 2017, 305:1-11.

19. Khan KS, Mack R, Castillo X, Kaiser M, Joergensen RG: Microbial biomass, fungal and bacterial residues, and their relationships to the soil organic matter C/N/P/S ratios. Geoderma 2016, $271: 115-123$.

20. Rath KM, Maheshwari A, Rousk J: The impact of salinity on the microbial response to drying and rewetting in soil. Soil Biology and Biochemistry 2017, 108:17-26.

21. Wichern J, Wichern F, Joergensen RG: Impact of salinity on soil microbial communities and the decomposition of maize in acidic soils. Geoderma 2006, $137(1-2): 100-108$.

22. Yan N, Marschner P, Cao W, Zuo C, Qin W: Influence of salinity and water content on soil microorganisms. International Soil and Water Conservation Research 2015, 3(4):316-323.

23. Li X, Sun M, Zhang H, Xu N, Sun G: Use of mulberry-soybean intercropping in salt-alkali soil impacts the diversity of the soil bacterial community. Microb Biotechnol 2016, 9(3) :293-304.

24. Chu H, Wang S, Yue H, Lin Q, Hu Y, Li X, Zhou J, Yang Y: Contrasting soil microbial community functional structures in two major landscapes of the $\mathrm{T}$ ibetan alpine meadow. MicrobiologyOpen 2014, $3(5): 585-594$. 
25. Qi Q, Zhao M, Wang S, Ma X, Wang Y, Gao Y, Lin Q, Li X, Gu B, Li $\mathrm{G}$ : The biogeographic pattern of microbial functional genes along an altitudinal gradient of the Tibetan pasture. Frontiers in microbiology 2017, 8:976.

26. Chen FH, Dong GH, Zhang DJ, Liu XY, Jia X, An C-B, Ma MM, Xie YW, Barton L, Ren X: Agriculture facilitated permanent human occupation of the Tibetan Plateau after 3600 BP. Science 2015, 347 (6219) :248-250.

27. Fan R, Short MD, Zeng S-J, Qian G, Li J, Schumann RC, Kawashima N, Smart RSC, Gerson AR: The formation of silicate-stabilized passivating layers on pyrite for reduced acid rock drainage. Environmental Science \& Technology 2017, 51 (19):11317-11325.

28. Nordstrom DK: Acid rock drainage and climate change. Journal of Geochemical Exploration 2009, $100(2-3): 97-104$.

29. Weatherill JJ, Krause S, Ullah S, Cassidy NJ, Levy A, Drijfhout FP, Rivett M0: Revealing chlorinated ethene transformation hotspots in a nitrate-impacted hyporheic zone. Water research 2019, $161: 222-231$.

30. Bolger AM, Lohse M, Usadel B: Trimmomatic: a flexible trimmer for I1lumina sequence data. Bioinformatics 2014, 30(15):2114-2120.

31. Truong DT, Franzosa EA, Tickle TL, Scholz M, Weingart G, Pasolli E, Tett A, Huttenhower C, Segata N: MetaPh1An2 for enhanced metagenomic taxonomic profiling. Nature methods 2015, $12(10): 902-903$.

32. Oksanen J, Kindt R, Legendre P, 0’ Hara B, Stevens MHH, Oksanen MJ, Suggests M: The vegan package. Community ecology package 2007, $10(631-637): 719$.

33. Goslee SC, Urban DL: The ecodist package for dissimilarity-based analysis of ecological data. Journal of Statistical Software 2007, $22(7): 1-19$.

34. Nurk S, Meleshko D, Korobeynikov A, Pevzner PA: metaSPAdes: a new versatile metagenomic assembler. Genome research 2017, $27(5): 824-834$.

35. Seemann T: Prokka: rapid prokaryotic genome annotation. Bioinformatics 2014, 30(14):2068-2069.

36. Li H, Durbin R: Fast and accurate long-read alignment with Burrows - Wheeler transform. Bioinformatics 2010, 26(5) :589-595.

37. Khorsandi F, Yazdi FA: Estimation of Saturated Paste Extracts' Electrical Conductivity from 1:5 Soil/Water Suspension and Gypsum. Communications in Soil Science and Plant Analysis 2011, $42(3): 315-321$. 
38. Kanso S, Patel BK: Microvirga subterranea gen. nov., sp. nov., a moderate thermophile from a deep subsurface Australian thermal aquifer. International journal of systematic and evolutionary microbiology 2003, $53(2)$ :401-406.

39. Martineau C, Mauffrey F, Villemur R: Comparative analysis of denitrifying activities of Hyphomicrobium nitrativorans, Hyphomicrobium denitrificans, and Hyphomicrobium zavarzinii. Applied and Environmental Microbiology 2015, 81 (15) :5003-5014.

40. Sauder LA, Albertsen M, Engel K, Schwarz J, Nielsen PH, Wagner M, Neufeld JD: Cultivation and characterization of Candidatus Nitrosocosmicus exaquare, an ammonia-oxidizing archaeon from a municipal wastewater treatment system. The ISME journal 2017, $11(5): 1142-1157$.

41. Shi Y, Delgado-Baquerizo M, Li Y, Yang Y, Zhu Y-G, Peñuelas J, Chu $\mathrm{H}$ : Abundance of kinless hubs within soil microbial networks are associated with high functional potential in agricultural ecosystems. Environment International 2020, 142:105869.

42. Hügler M, Sievert SM: Beyond the Calvin cycle: autotrophic carbon fixation in the ocean. Annual review of marine science 2011, 3:261-289.

43. Bar-Even A, Noor E, Milo R: A survey of carbon fixation pathways through a quantitative lens. Journal of experimental botany 2012, $63(6): 2325-2342$.

44. Crecchio C, Gelsomino A, Ambrosoli R, Minati JL, Ruggiero P: Functional and molecular responses of soil microbial communities under differing soil management practices. Soil Biology and Biochemistry 2004, 36(11):1873-1883.

45. Tyson GW, Chapman J, Hugenholtz P, Allen EE, Ram RJ, Richardson PM, Solovyev VV, Rubin EM, Rokhsar DS, Banfield JF: Community structure and metabolism through reconstruction of microbial genomes from the environment. Nature 2004, 428 (6978):37-43.

46. Dahl C, Franz B, Hensen D, Kesselheim A, Zigann R: Sulfite oxidation in the purple sulfur bacterium Allochromatium vinosum: identification of SoeABC as a major player and relevance of SoxYZ in the process. Microbiology 2013, 159 (Pt_12):2626-2638.

47. Pott AS, Dahl C: Sirohaem sulfite reductase and other proteins encoded by genes at the dsr locus of Chromatium vinosum are involved in the oxidation of intracellular sulfur. Microbiology 1998, $144(7): 1881-1894$.

48. Zhu $Y-G$, Williams PN, Meharg AA: Exposure to inorganic arsenic from rice: a global health issue? Environmental pollution 2008, 154 (2) : 169-171. 
49. Thoma F: Light and dark in chromatin repair: repair of UV - induced DNA lesions by photolyase and nucleotide excision repair. The EMBO journal 1999, 18 (23):6585-6598.

50. Ermolenko D, Makhatadze G: Bacterial cold-shock proteins. Cellular and Molecular Life Sciences CMLS 2002, 59(11):1902-1913.

51. Morgan-Kiss RM, Priscu JC, Pocock T, Gudynaite-Savitch L, Huner NP: Adaptation and acclimation of photosynthetic microorganisms to permanently cold environments. Microbiology and molecular biology reviews 2006, 70(1):222-252.

52. Fujii DK, Fulco AJ: Biosynthesis of unsaturated fatty acides by bacilli. Hyperinduction and modulation of desaturase synthesis. [Bacillus megaterium]. J Biol Chem; (United States) 1977, 252 (11).

53. Zhu K, Choi KH, Schweizer HP, Rock CO, Zhang YM: Two aerobic pathways for the formation of unsaturated fatty acids in Pseudomonas aeruginosa. Molecular microbiology 2006, 60(2):260-273.

54. Chen $L^{-x}$, Hu M, Huang $L-n$, Hua $Z^{-}$, Kuang J-1, Li S-j, Shu W-s: Comparative metagenomic and metatranscriptomic analyses of microbial communities in acid mine drainage. The ISME journal 2015, 9(7) : 1579-1592.

55. Baker-Austin C, Dopson M: Life in acid: $\mathrm{pH}$ homeostasis in acidophiles. Trends in microbiology 2007, 15(4):165-171.

56. Wang M, Chen S, Chen L, Wang D: Responses of soil microbial communities and their network interactions to saline-alkaline stress in Cd-contaminated soils. Environmental Pollution 2019, 252:1609-1621. 
Figures
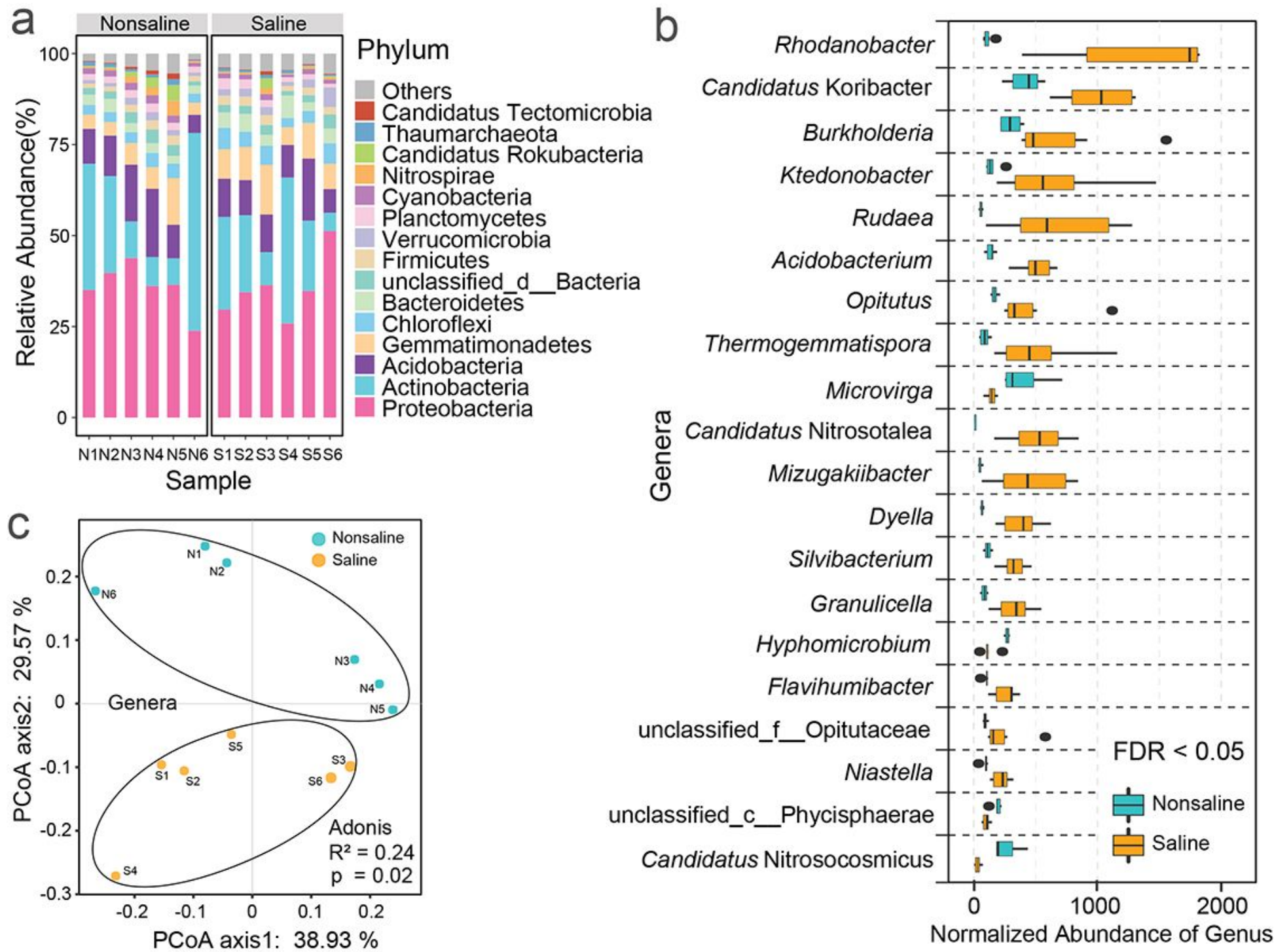

Normalized Abundance of Genus

Figure 1

Composition and differences in saline and nonsaline soil on the Tibetan Plateau. a. Bacterial composition at the phylum level in saline and nonsaline soil samples of Tibetan Plateau. b. Top 20 genera with significant differences (FDR < 0.05) in saline (orange) and nonsaline (cyan) soil samples of Tibetan Plateau, the total number of reads is normalized to 100000 . c. Principal coordinate analysis of saline (orange) and nonsaline (cyan) soil samples based on the composition and abundances of the bacterial communities at the genus level. 


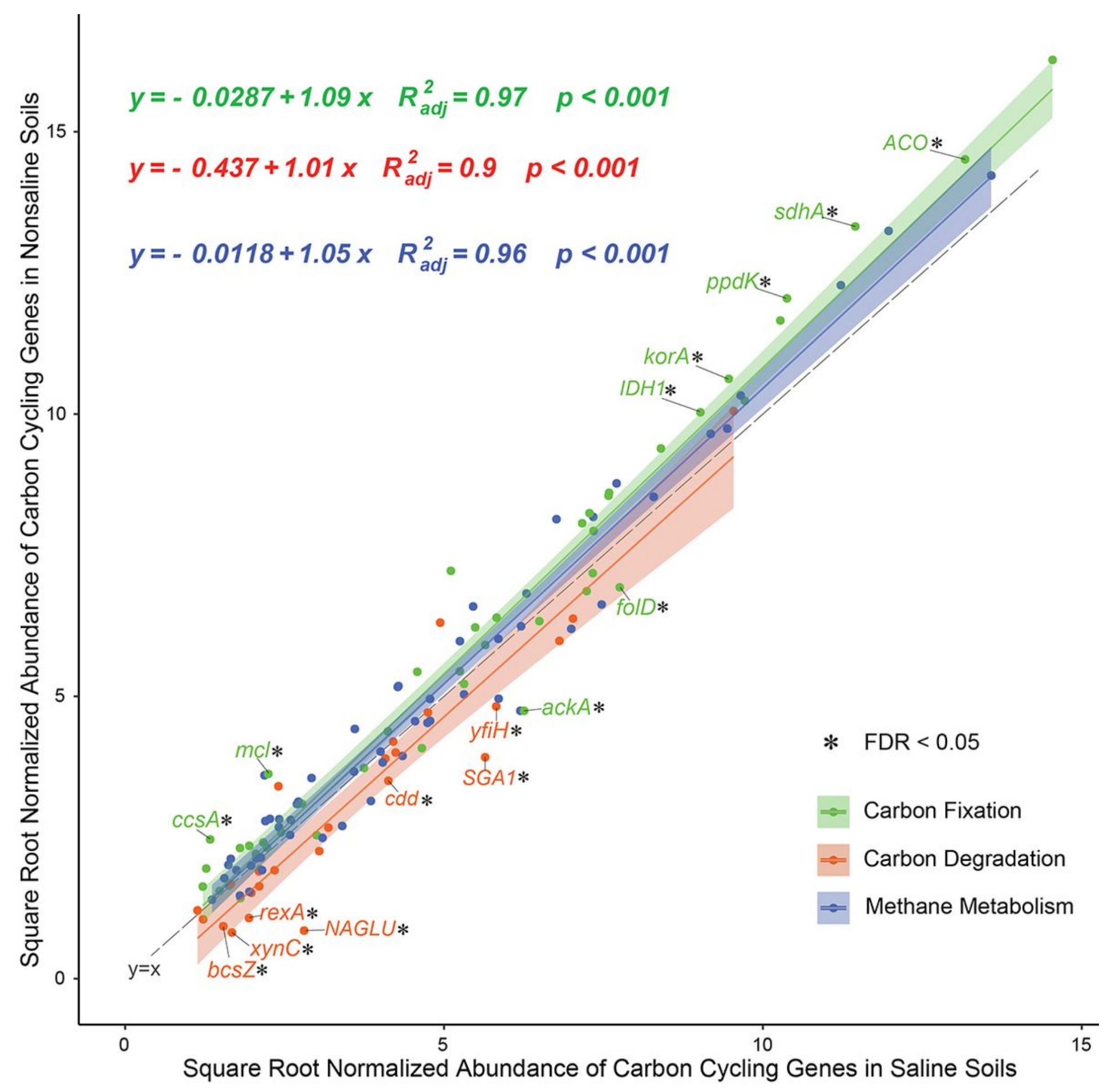

Figure 2

The ratio of genes in carbon cycling pathway in saline and nonsaline soil on the Tibetan Plateau. The black dotted line equation " $y=x$ " indicates that the horizontal and vertical axes are equal. Green, red, and blue represent genes of carbon fixation, carbon degradation, and methane metabolism pathways, respectively. Genes with significant differences $(F D R<0.05)$ are marked in the corresponding colors and connected with short lines. The total number of reads is normalized to 100000. 


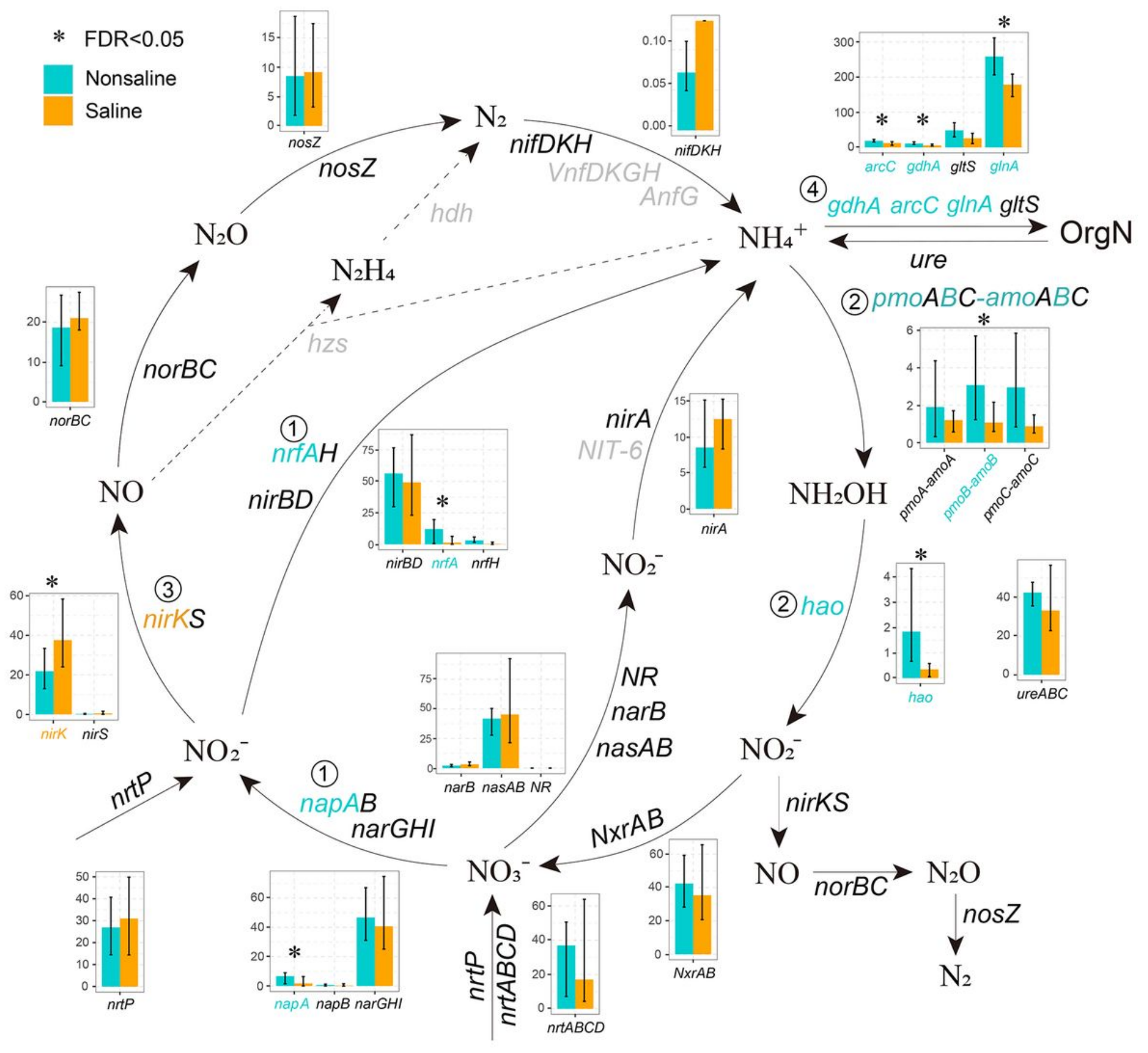

Figure 3

Differences in the abundance of genes related to nitrogen cycling in the saline (orange) and nonsaline (cyan) soil on the Tibetan Plateau. Bar plots show the normalized abundances of nitrogen cycling genes. Significantly different $(F D R<0.05)$ genes are marked with " " and circled numbers. Undetected genes are indicated in gray. Circled numbers identify genes with significant differences: 1, dissimilatory nitrate reduction; 2 , nitrification; 3 , denitrification; and 4 , organic nitrogen conversion. The total number of reads is normalized to 100000 . 


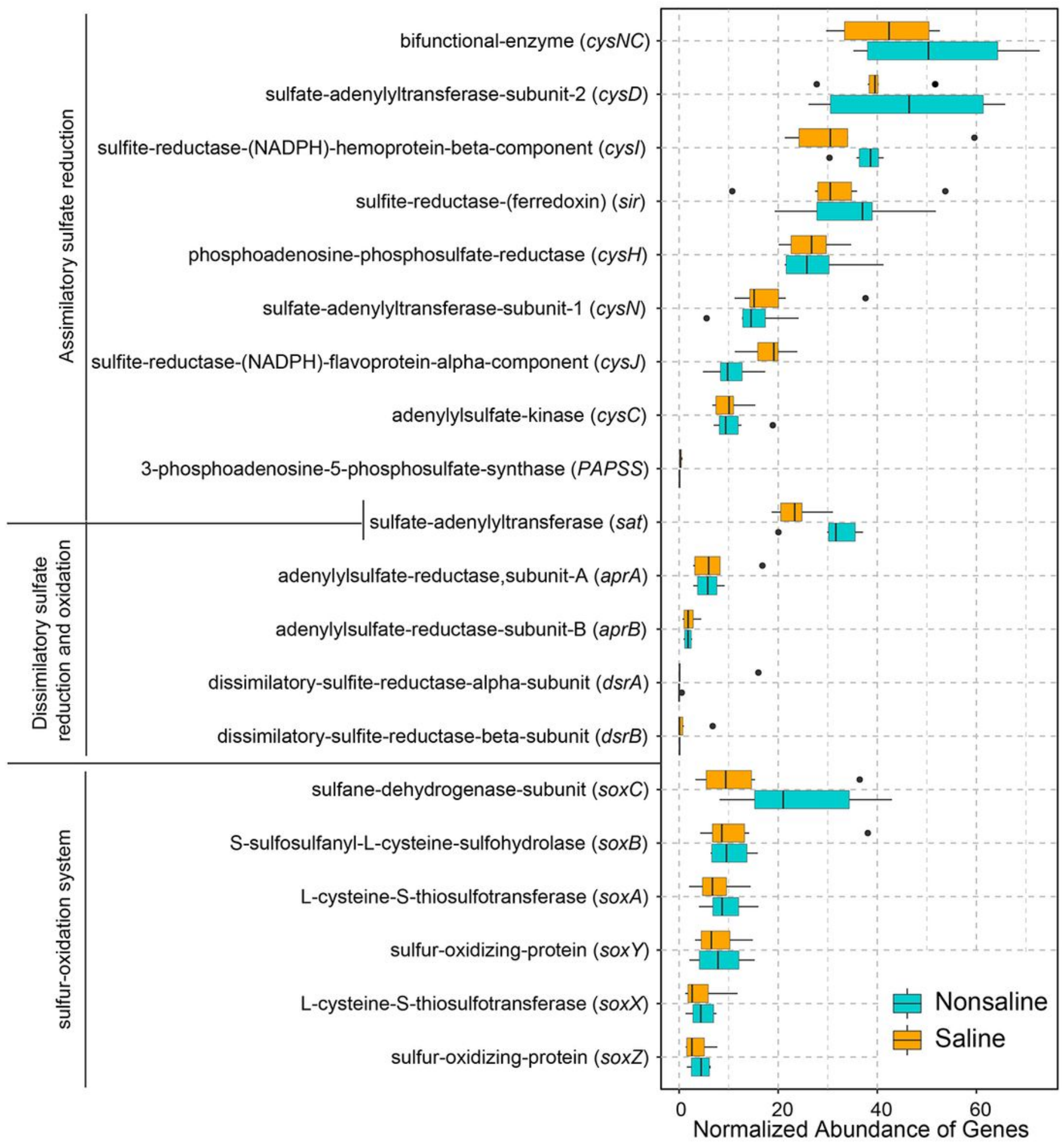

Figure 4

Difference in abundances of sulfur cycling genes in saline (orange) and nonsaline (cyan) soil on the Tibetan Plateau. The total number of reads is normalized to 100000. 


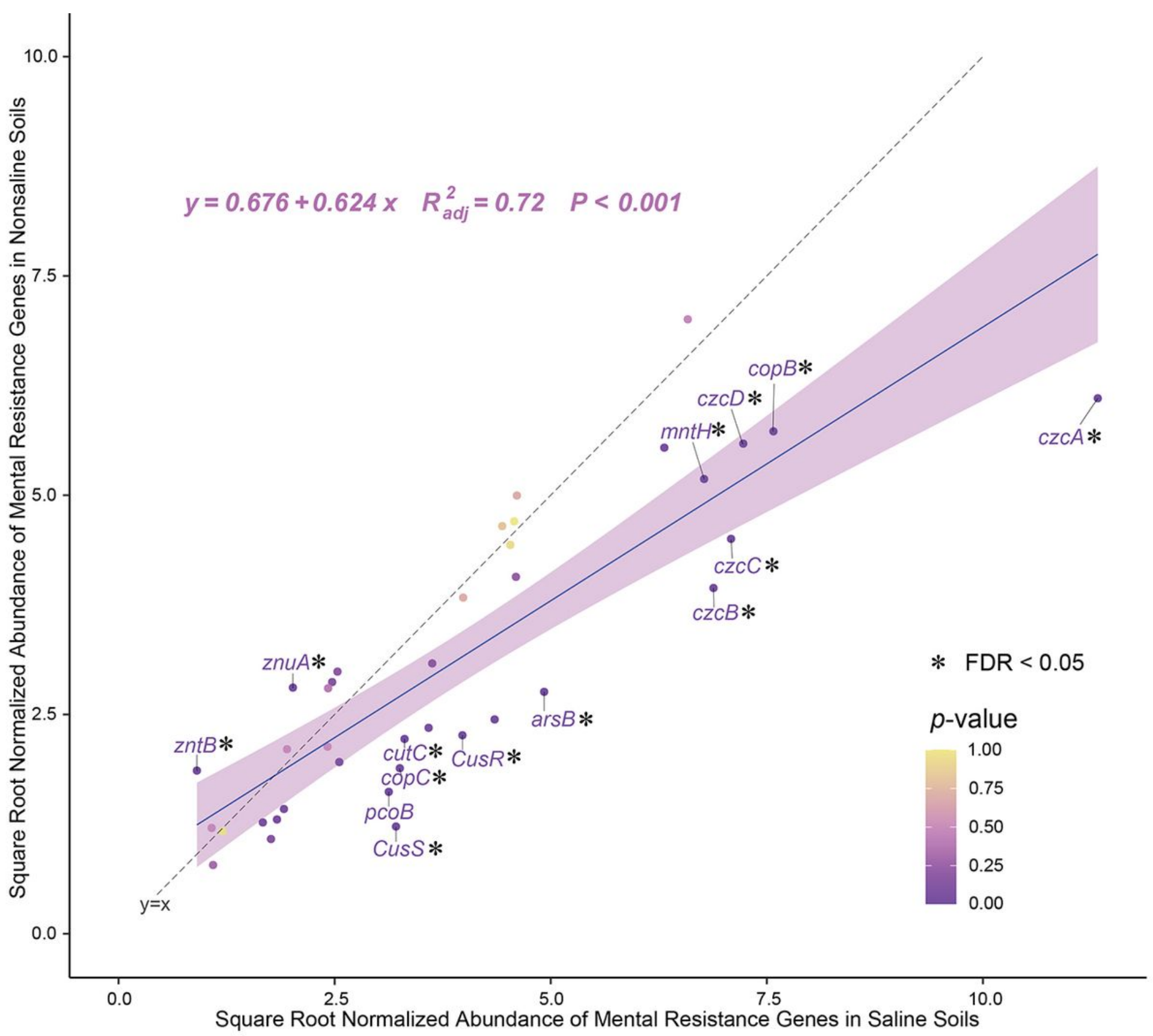

\section{Figure 5}

Abundance of heavy metal(loid) resistance genes in saline and nonsaline soil on the Tibetan Plateau. The black dotted line with the equation " $y=x$ " indicates that the horizontal and vertical axes are equal. Genes with significantly different abundances $(F D R<0.05)$ are marked in purple and connected with short lines. The total number of reads is normalized to 100000 . 


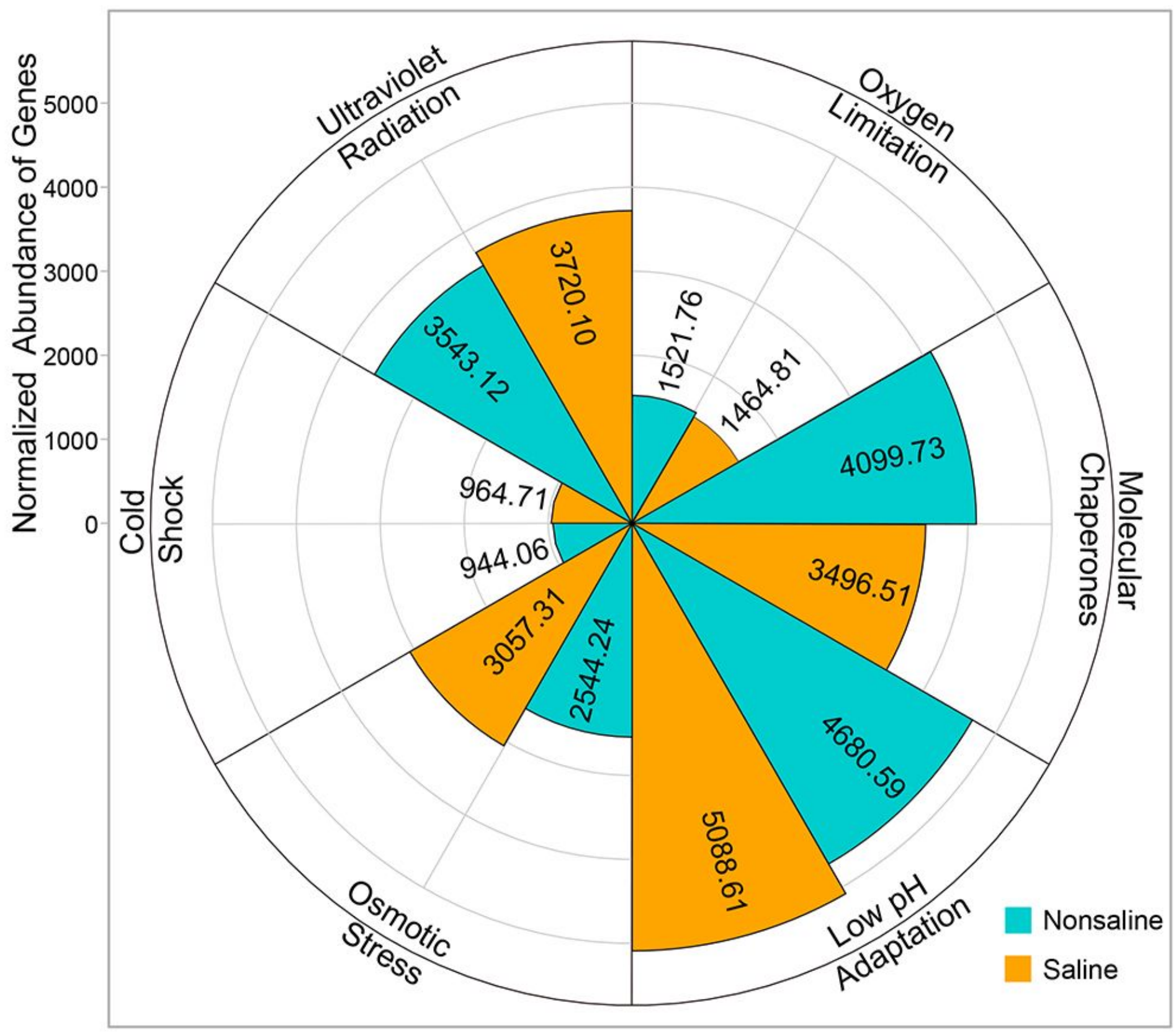

Figure 6

Abundance of the environmental stress resistance genes. The total number of reads is normalized to 100000. 


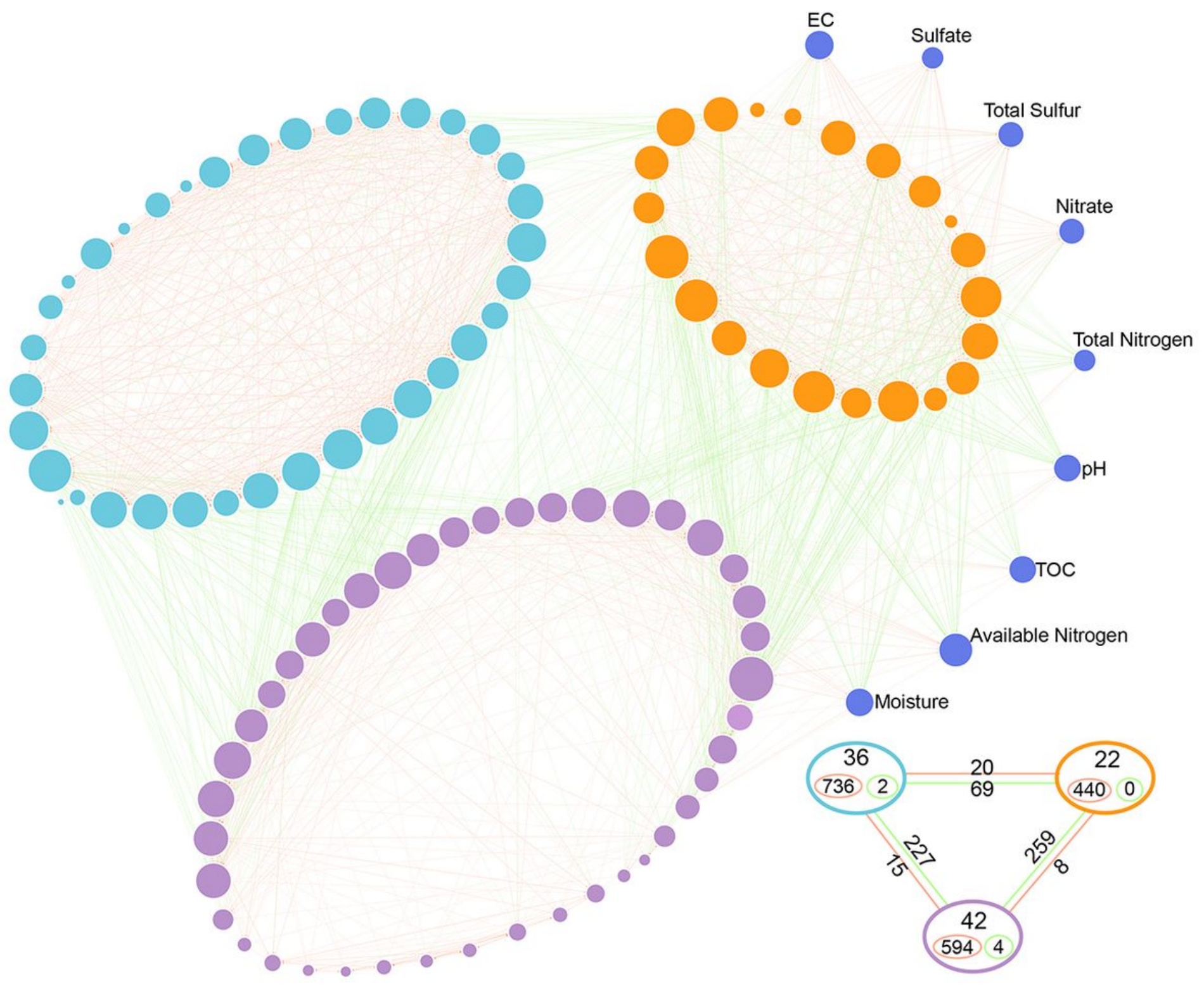

Figure 7

Network of the top 100 dominant genera and physicochemical parameters. A connection denotes a Spearman's correlation coefficient with a magnitude greater than 0.6 (positive correlation $=$ red lines) or less than -0.6 (negative correlation $=$ green lines) and statistically significant ( $p$-value $<0.05)$. The size of each node is proportional to the number of connections (i.e., degree). The thickness of each connection between two nodes (i.e., edge) is proportional to the Spearman's correlation coefficient (i.e., weight), ranging from $|0.6|$ to $|1|$. The network is colored according to the modules, where the nodes clustered in the same module share the same color. The lower right corner is a schematic diagram, and the large circles represent three modules based on network analysis, and the positions are corresponding. In the large circles, the top number indicates the number of nodes, the red circle and the number indicate positive correlation within the module, and the green indicates negative correlation. Between the large circles, red lines and numbers indicate positive correlations, and green lines and numbers indicate negative correlations. 


\section{Supplementary Files}

This is a list of supplementary files associated with this preprint. Click to download.

- supplementary1.docx 